\title{
Ultrahigh-Speed TV Commercial Detection, Extraction, and Matching
}

\author{
Xiaomeng $\mathrm{Wu}^{*}$, Member, IEEE, and Shin'ichi Satoh, Member, IEEE
}

\begin{abstract}
We describe a system based on exact-duplicate matching for detecting and localizing TV commercials in a video stream, clustering the exact duplicates, and detecting duplicate exact-duplicate clusters across video streams. A twostage temporal recurrence hashing algorithm is used for the detection, localization, and clustering. The algorithm is fully unsupervised, generic, and ultrahigh speed. Another algorithm is used to integrate the video and audio streams to achieve higher performance extraction. Its sequence- and frame-level accuracies in testing were respectively $98.1 \%$ and $97.4 \%$. A third algorithm uses a new bag-of-fingerprints model to detect duplicate exactduplicate clusters across multiple streams. It is robust against decoding errors. The contributions include: 1) fully unsupervised detection, extraction, and matching of exact duplicates; 2) more generic commercial detection than with the knowledge-based techniques; 3) ultrahigh-speed processing, which detected the TV commercials from a 1-month video stream in less than 42 minutes, which is more than ten times faster than with state-ofthe-art algorithms; 4) more generic operation in terms of signal input, the performance of which is consistent between video and audio streams. Testing using a video database containing a 10hour, a 1-month, and a 5-year video stream comprehensively demonstrated the effectiveness and efficiency of this system.
\end{abstract}

Index Terms-Content-based retrieval, data mining, search methods, video signal processing.

\section{INTRODUCTION}

$\mathbf{T}$ $\mathrm{V}$ commercials, also known as commercial films, are a form of communication intended to persuade an audience to consume or purchase a particular product or service. They are generally considered the most effective means of massmarket advertising. Although TV commercials are usually considered to be an annoyance, their extraction based on detection and localization is an important preprocessing step in broadcast media analysis. Their evaluation is important not only for competitive marketing analysis but also for advertising planning and as a barometer of the advertising industry's health.

TV commercial extraction systems are used to detect and extract the commercials in one or more video streams. Here, "detection" indicates discovering the existence of commercials from the video streams, while "extraction" or "localization" indicates exactly locating the beginning and end positions of a commercial. TV commercial extraction has thus attracted substantial research attention recently, and extensive effort has gone into developing techniques for detecting and localizing TV commercials broadcast on different channels at different times. An important application of TV commercial extraction is to video mining systems, which could be used to categorize, filter, and recommend commercials on the basis of personalized preferences [1].
TV commercial extraction techniques can be categorized as knowledge-based and repetition-based. The knowledgebased ones [2]-[7] use the intrinsic characteristics of TV commercials (e.g. the presence of monochrome, silence, or product frames) as a priori knowledge for detecting them. Most knowledge-based techniques are efficient but not generic enough because of the data-dependent a priori knowledge they use. For example, most Japanese TV stations do not use monochrome or silence frames to separate consecutive TV commercials. Other characteristics (e.g. product frame, shot information, and motion information) normally vary by country and sometimes by region. This makes it difficult to construct a generic TV commercial detection system that works equally well for the TV commercials of various countries.

The repetition-based techniques [8]-[16] are based on a particular property of TV commercials: there are usually exact duplicates of each one in a sufficiently long video stream. While repetition-based techniques are unsupervised and more generic, they impose a large computational burden. The amount of computation with knowledge-based techniques usually increases linearly with the size of the video archive while that of repetition-based ones increases more than linearly. How to minimize the cost of each comparison to enable the application of repetition-based techniques to a large-scale archive is one of the main issues that have to be addressed with these techniques.

We have developed a repetition-based system for ultrahighspeed exact-duplicate TV commercial detection, extraction, and matching. A two-stage temporal recurrence hashing algorithm (TRHA) is used to detect and localize the exactduplicate commercials. The exact duplicates are detected on the basis of the temporal consistency of their recurring frame alignment. In an experiment, it was able to detect and localize the exact-duplicate commercials in a 1-month video streams in less than 42 minutes, more than ten times faster than with conventional brute-force matching of recurring fragment pairs. This is the fastest detection and localization reported for a fully unsupervised algorithm.

A second algorithm is used to integrate the video and audio streams to achieve higher performance exact-duplicate extraction. Its sequence- and frame-level accuracies in testing were both about $98 \%$.

In large-scale application, the video archive is divided into multiple video streams of a certain length such that the computational effort and memory usage needed for processing each stream are low enough for practical use. The multiple streams are processed in parallel to reduce the overall processing time. The exact duplicates across the streams must 
be matched and grouped (i.e., clustered) in order to obtain the final set of duplicate commercials. For this matching task, we developed a third algorithm that uses a new bag-offingerprints (BOFs) model. In our experiment, this algorithm effectively detected duplicate exact-duplicate clusters across multiple video streams and was robust against decoding and fingerprinting errors.

In this work, we

- aim at fully unsupervised exact-duplicate detection, extraction, and matching of TV commercials; i.e. neither a training database nor a reference dataset is provided beforehand.

- aim at more generic exact-duplicate commercial detection than with the knowledge-based techniques; i.e. little a priori knowledge is used in our detection, localization, and extraction algorithm.

- assume that the boundaries of commercials are unknown and thus aim at automatic detection, localization, and clustering of TV commercials broadcast on different channels at different times, the three capabilities mentioned above.

- aim at ultrahigh-speed exact-duplicate detection, localization, clustering, and matching.

- aim at more generic operation in terms of signal input; i.e. performance is consistent between video and audio streams.

The remainder of this paper is organized as follows. Section II overviews related work. Section III formulates the exact-duplicate detection and localization problem, discusses previously reported methods for solving this problem, and describes our two-stage TRHA for TV commercial detection, localization, and clustering and our algorithm for video and audio stream integration. Section IV describes the algorithm used for cross-stream exact-duplicate cluster matching and grouping, including the criteria used for the matching. Section V describes our experimental evaluation. Section VI discusses the application of our system to TV commercial mining for knowledge rediscovery. Section VII summarizes the key points and mentions future work.

\section{RELATED WORK}

\section{A. Knowledge-Based TV Commercial Extraction}

Knowledge-based TV commercial extraction techniques focus on the intrinsic characteristics of TV commercials and use them as a priori knowledge for detecting them. These characteristics include monochrome frames [2], [3], [6], [7], silence frames [3], [5], [6], product frames [4], [6], duration [7], shot information (e.g. frequency or style of cuts) [2]-[5], and motion information (e.g. edge change ratio, motion vector length) [2], [3], [5], [7]. Some of these techniques [2], [3], [7] apply heuristic rules to the characteristics to distinguish commercials from regular programs. Others [4]-[6] attempt to train a binary TV commercial classifier created by machine learning. Knowledge-based techniques are more efficient than repetition-based ones but not generic enough because of the data-dependent a priori knowledge they use.

\section{B. Repetition-Based TV Commercial Extraction}

Because a single TV commercial can be broadcast repeatedly over the course of weeks, months, and even years, TV commercials are characterized by their repetition in a broadcast video stream. Several recently reported methods use this property to detect commercials. In this approach, a TV commercial is considered an instance of an original without any transformation. The process involves two stages: 1) searching for recurring frames or frame fragments and 2) detecting the recurring ones as duplicate instances on the basis of their temporal consistency.

For the first stage, most extraction methods [8], [9], [11], [13], [15]-[17] apply a frame fingerprinting technique to all frames in a video stream and insert each frame into a hash table with the corresponding fingerprint considered to be an inverted index. An important assumption underlying these methods is that the recurring frames of exact duplicate instances have exactly the same fingerprint, so a hash collision occurs in the hash bucket corresponding to these frames. This means that the recurring frames of exact duplicates can be efficiently detected by using a collision attack process. This hashingbased technique has been considered a state-of-the-art choice for extraction, but it is memory intensive. Methods that use classic retrieval [10], [12] or clustering [14] to tackle this problem are more computationally intensive than hashing.

For the second stage, the temporal consistency constraints between the recurring frames detected in the first stage are used to detect and localize duplicate instances. Some methods [14]-[16] do this by applying brute-force pairwise matching to the recurring frames on the basis of their temporal consecutiveness. Haitsma and Kalker [17] proposed applying a temporal growing strategy, instead of pairwise matching, to each pair of recurring frames. These methods are discussed in Section III-D in more detail.

Repetition-based techniques are unsupervised and more generic than knowledge-based ones but impose a larger computational burden. From the TV commercial extraction viewpoint, another disadvantage is the lower discriminative power for distinguishing TV commercials from non-commercial duplicates. This disadvantage can be easily compensated for by using a post-processing strategy. The restricted duration of TV commercials is one of the most useful pieces of a priori knowledge for this purpose [14]-[16].

\section{Near-Duplicate Detection}

In addition to exact-duplicate detection, there is another research direction known as near-duplicate detection. Near duplicates are approximately identical videos. They are derived from an original video, usually by means of various transformations including cam-cording, picture-in-picture insertion, pattern insertion, strong re-encoding, gamma correction, and quality adjustment. As described above, the recurring frames of exact duplicates usually cause a hash collision if they are detected using fingerprinting and hashing techniques. This does not happen when detecting near duplicates due to the transformations made to them. 
Techniques for near-duplicate detection can be categorized as image-based and sequence-based. The image-based ones [18]-[21] focus on keyframes sampled from the video stream rather than all frames. They use image duplicate detection or image retrieval techniques, including the use of spatially local descriptors and a bag-of-words model, to detect duplicate images. Some of them [19]-[21] exploit the weak geometric consistency constraints between keyframes to penalize the local descriptors that are not consistent in terms of affine transformation. These techniques are highly effective for detecting duplicate images, but they generate only coarse temporal keyframe alignment, which is not adequate for precise localization of duplicate images. The sequencebased techniques [22]-[25] embed temporal consistency constraints between near duplicate images during the detection process to address this problem. They exploit spatiotemporal information on the basis of local point trajectories [22], frame sequences [23], and video cuboids [24], [25].

The near-duplicate detection techniques have superior performance in tackling severe transformation problems compared to the exact-duplicate ones. The main reason for this lies in their usage of local features for describing the image or frame sequences. These features are much more informative than fingerprints. However, the high memory usage and computational effort imposed make these techniques unsuitable for large-scale archives. Frame sampling, e.g. keyframe selection, is usually used as a solution to this problem, but this reduces the accuracy of the temporal localization of duplicates.

\section{Duplicate Detection vs. Duplicate Extraction}

Duplicate-instance commercial extraction techniques can also be categorized from the task-setting viewpoint. In some studies [9], [13], [17]-[26], the task was to determine for each query in a reference set of queries the location at which the query occurs in a test collection of video streams. The techniques used in these studies were not fully unsupervised considering the construction of the reference set. Moreover, the boundaries of the duplicate videos in the reference set had to be known beforehand, which is not realistic and thus further enlarges the workload. Thanks to the availability of the reference set, the computational cost increases only linearly with the size of the test collection. A more challenging task was addressed in other studies [8], [10]-[12], [14]-[16]: determine the locations, if any, at which duplicate instances occur in a test collection of video streams. This is the main problem addressed in this paper. Neither a reference database nor a video-clip boundary database is provided beforehand. The system should be fully unsupervised, and the algorithms should minimize the cost of each individual operation because the amount of computation increases with the square of the test collection size.

\section{INDIVIDUAL-STREAM EXACT-DUPLICATE DETECTION AND LOCALIZATION}

\section{A. System Overview}

We adopted the repetition-based approach for the TV commercial detection and extraction task because the results so far are more generic than with the knowledge-based one. We did not use the near-duplicate detection framework because its usage of local feature leads to high memory usage and high computation cost. TV commercials are regarded here as exact duplicates because they are broadcast repeatedly without any transformation. As described in Section II-B, the exactduplicate extraction process involves two stages: 1) searching for recurring frames or frame fragments and 2) detecting the recurring ones as duplicate instances on the basis of their temporal consistency. The first task can be quickly done by using frame hashing, and the second can be done using a state-of-the-art method exploiting temporal consistency, but this imposes a large computational burden.

Given a large-scale video stream as input, the task is to detect and cluster exact-duplicate videos. For the first stage, we use the classic solution, i.e. frame fingerprinting and hashing, for detecting recurring video fragments. A video fragment is a short segment composed of consecutive and fingerprintidentical frames. It is similar to but much shorter than a shot or sub-shot. A video stream can be regarded as a very long alphabetic string, in which each letter corresponds to a fragment in the stream. A set of fingerprints is regarded as set of letters, in which each letter corresponds to a fingerprint. The exact-duplicate extraction task can thus be formulated into a string extraction problem: given a long string of letters as the input, the task is to detect clusters of substrings such that each pair of substrings in a cluster form an approximate match.

For the second stage, we exploit the temporal alignment of the recurring letters detected in the first stage. That is, two substrings are considered to be duplicates if the temporal structures of their recurring letters are approximately consistent with each other. In contrast to studies that used pairwise matching [15], clustering [14], [16], or frame growing [17], we use a second-stage hashing algorithm. For each pair of recurring letters, two hashing functions are defined for capturing their temporal geometry. The potential temporal consistency of letter alignment is detected by inserting all recurring letter pairs into a two-dimensional hash table and searching for hash collisions. Letter pairs colliding in a hash bin are assembled to form candidates of approximate matches. Morphological filtering is conducted to remove the clutter and to adequately determine the boundaries of the duplicate substrings. Finally, transitive closure is conducted to combine duplicate substrings into clusters. Table I lists the notations used in this section.

\section{B. Frame Fingerprinting and Hashing}

A number of studies have applied frame fingerprinting and hashing techniques to exact-duplicate extraction. The fingerprinting has been based on luminance moments [16], [27], color moments [8], [11], [13], a color histogram [9], a gradient histogram [15], an ordinal signature [9], and acoustic signatures [17], [28]. To achieve performance consistency between video and audio streams, we chose a fingerprinting strategy based on luminance moments [27] for video hashing because of its simplicity and effectiveness. After intensity normalization, each video frame is partitioned into $4 \times 4$ blocks, as shown in Figure 1a. The first and second luminance 
TABLE I

EXPLANATION OF NOTATIONS USED IN SECTION III.

\begin{tabular}{|c|c|}
\hline Notation & Explanation \\
\hline$\Phi$ & string formulated from video stream \\
\hline$\phi_{i}$ & $i$ th letter in string $\Phi$ \\
\hline$\Psi$ & $\begin{array}{l}\text { finite ordered alphabet containing letters occurring in } \\
\text { the string } \Phi\end{array}$ \\
\hline$\psi$ & $\begin{array}{l}\text { letter, e.g. 'W' and 'O' occurring in the string shown in } \\
\text { Figure } 2\end{array}$ \\
\hline$\phi_{i} \cdots \phi_{j}$ & $\begin{array}{l}\text { substring beginning at } i \text { th frame and ending at } j \text { th frame } \\
\text { in the string } \Phi\end{array}$ \\
\hline$\hat{\Psi}$ & $\begin{array}{l}\text { finite ordered alphabet containing letters occurring at } \\
\text { least twice in the string } \Phi\end{array}$ \\
\hline $\operatorname{occ}(\psi)$ & $\begin{array}{l}\text { set containing the positions at which letter } \psi \text { occurs in } \\
\Phi\end{array}$ \\
\hline$()$, & $\begin{array}{l}\text { letter recurrence, e.g. }(9,18) \text {, in which each element } \\
\text { corresponds to a temporal position }\end{array}$ \\
\hline $\operatorname{rec}(\psi)$ & set containing recurrences of letter $\psi$ \\
\hline $\operatorname{REC}(\Phi)$ & $\begin{array}{l}\text { set containing all letter recurrences derived from the } \\
\text { string } \Phi\end{array}$ \\
\hline$\delta$ & $\begin{array}{l}\text { restricted length of the duplicate instance to be detected; } \\
\text { used as a threshold in both Döhring's method and our } \\
\text { proposed algorithm }\end{array}$ \\
\hline AdjMat & $\begin{array}{l}\text { two-dimensional adjacency matrix, which is output of } \\
\text { both methods of Döhring and Lienhart and Berrani et } \\
\text { al. }\end{array}$ \\
\hline $\operatorname{sim}()$ & $\begin{array}{l}\text { similarity between two occurrence sets, which is used } \\
\text { in the method of Berrani et al. }\end{array}$ \\
\hline$\sigma$ & $\begin{array}{l}\text { threshold of similarity between two occurrence sets, } \\
\text { which is used in the method of Berrani et al. }\end{array}$ \\
\hline HamDis() & $\begin{array}{l}\text { Hamming distance between two substrings, which is } \\
\text { used in Haitsma and Kalker's method }\end{array}$ \\
\hline$\eta$ & $\begin{array}{l}\text { threshold of the Hamming distance between two sub- } \\
\text { strings, which is used in Haitsma and Kalker's method }\end{array}$ \\
\hline$h_{1}(i, j)$ & $\begin{array}{l}\text { recurrence hash function corresponding to temporal } \\
\text { position of previous occurrence of letter recurrence } \\
(i, j)\end{array}$ \\
\hline$h_{2}(i, j)$ & $\begin{array}{l}\text { recurrence hash function corresponding to temporal } \\
\text { interval between two occurrences of letter recurrence } \\
(i, j)\end{array}$ \\
\hline$r_{1}$ & resolution factor of hash function $h_{1}()$ \\
\hline$r_{2}$ & resolution factor of hash function $h_{2}()$ \\
\hline$(;)$ & $\begin{array}{l}\text { pair of recurrence hash values, e.g. (1;9) indicating } \\
h_{1}()=1 \text { and } h_{2}()=9\end{array}$ \\
\hline
\end{tabular}

moments are computed for each block to generate a 32dimensional $(4 \times 4 \times 2)$ vector. The vector is further binarized in a 32-bit fingerprint by comparing each component with the average of the corresponding moment. For the audio hashing, we chose the acoustic signature proposed by Haitsma and Kalker [17], which has been demonstrated to be robust and reliable in the content-based copy detection task of the Text Retrieval Conference Video Retrieval Evaluation (TRECVID) workshop [26]. It focuses on the energies of the 33 barkscale bands of each audio frame, as shown in Figure 1b, and uses the sign of the energy band differences both on the time and frequency axes as a 32-bit fingerprint. The frame rate of the two streams is 30 . We did not test all existing signatures, but we believe that any global feature with reasonable discriminative power can be used for this purpose.

It is noted that the reason why we use two input sources (video and audio) is to investigate the performance consistency of the proposed algorithm. The availability of both sources is not a must here. The input of the proposed algorithm can be

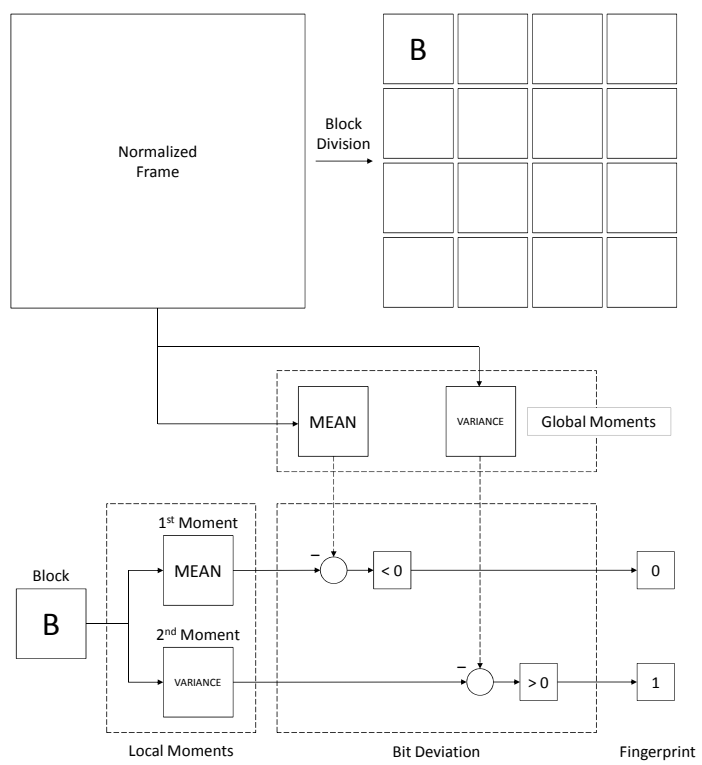

(a) Video fingerprinting

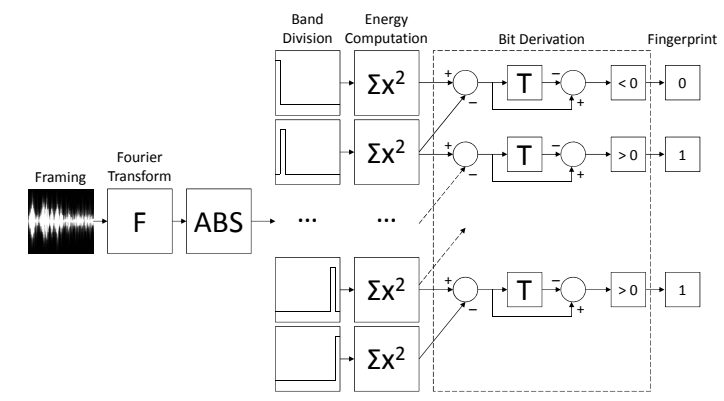

(b) Audio fingerprinting [17]

Fig. 1. Fingerprinting strategies used in this system.

video only, audio only, or both, which depends on the needs of the real applications.

Frames with the same fingerprint can be considered visually or acoustically identical. To minimize the search space, continuous frames with the same fingerprint are combined to form a video fragment. A fragment is similar to but much shorter than a shot or sub-shot. The video stream can thus be regarded as a very long string $\Phi$ of letters, in which each letter $\phi$ corresponds to a fragment in the video stream. The set of fingerprints is regarded as a finite ordered alphabet $\Psi$, in which each letter $\psi$ corresponds to a fingerprint. The exactduplicate commercial extraction task can thus be formulated into a string extraction problem: given a long string $\Phi$ of letters as the input, the task is to detect clusters of substrings so that each pair of substrings in a cluster form an approximate match.

A string $\Phi$ is a chain $\phi_{1} \cdots \phi_{n}$ of letters $\phi_{i} \in \Psi$. We let $|\Phi|=n$ denote the length of the string, and $|\Phi|=25$ for the example shown in Figure 2. We let $|\Psi|$ denote the size of the alphabet. In theory, $|\Psi| \leq 2^{m}$ with $m$ being the number of bits in the fingerprint, but practically, $|\Psi| \ll 2^{m}$ in most cases. For instance, $|\Psi|=16$ for the example in Figure 2. All letters in the string are then inserted into a hash table. The table has length $|\Psi|$ such that each letter $\psi \in \Psi$ maps to only 


\begin{tabular}{|c|c|c|c|c|c|c|c|c|c|c|c|c|c|c|c|c|c|c|c|c|c|c|c|c|}
\hline 0 & 1 & 2 & 3 & 4 & 5 & 6 & 7 & 8 & 9 & 10 & 11 & 12 & 13 & 14 & 15 & 16 & 17 & 18 & 19 & 20 & 21 & 22 & 23 & 24 \\
\hline W & O & R & D & S & C & U & T & M & O & R & E & T & H & A & N & S & W & O & R & D & S & X & Y & Z
\end{tabular}

Fig. 2. Example of string formulated from video stream. Number above each letter indicates corresponding temporal position.

one entry in the table. Each entry in the table points to a linked list storing the positions at which the associated letters occur in $\Phi$. Recurring letters can thus be detected by searching for hash collisions.

\section{Preliminaries}

We refer to a letter that occurs at least twice in string $\Phi$ as a 'recurring letter'. We let $\hat{\Psi}$ denote the alphabet, in which all the letters are recurring. $\hat{\Psi}$ is a subset of $\Psi$; it is equal to $\{$ 'W', 'O','R', 'D', 'S','T'\} for the example in Figure 2. For each recurring letter $\psi \in \hat{\Psi}$, we have the set of positions in $\Phi$ at which it occurs. This 'occurrence set of $\psi$ ' is defined as

$$
\operatorname{occ}(\psi):=\left\{i: \phi_{i}=\psi, 0 \leq i<|\Phi|\right\}
$$

For instance, $\operatorname{occ}\left({ }^{\prime} \mathrm{O}\right.$ ') $=\{1,9,18\}$ for the example in Figure 2. Given the occurrence set $\operatorname{occ}(\psi)$ of a letter $\psi$, each 2combination of $\operatorname{occ}(\psi)$ can be denoted as a pair of positions $(i, j)$ with $i \in \operatorname{occ}(\psi)$ and $j \in \operatorname{occ}(\psi)$. This pair corresponds to the positions of the temporal recurrences of $\psi$. We refer to this pair as the 'letter recurrence of $\psi$ '. Note that a letter recurrence differs from a recurring letter. A recurring letter is a letter occurring multiple times in a string, while a letter recurrence is the pair of positions where a recurring letter occurs in the string. For each recurring letter $\psi$, we thus have a set of letter recurrences. We refer to this set as the 'recurrence set of $\psi^{\prime}$ and define it as

$$
\operatorname{rec}(\psi):=\left\{(i, j): \phi_{i}=\phi_{j}=\psi, 0 \leq i<j<|\Phi|\right\}
$$

For instance, $\operatorname{rec}(' \mathrm{O}$ ') $=\{(1,9),(1,18),(9,18)\}$ for the example in Figure 2. We further combine the recurrence sets of all $\psi \in \hat{\Psi}$. This combination is referred to as the recurrence set of string $\Phi$ and denoted as $\operatorname{REC}(\Phi)$, which can be defined as

$$
\operatorname{REC}(\Phi):=\{\operatorname{rec}(\psi): \psi \in \hat{\Psi}\}
$$

or

$$
\operatorname{REC}(\Phi):=\left\{(i, j): \phi_{i}=\phi_{j}, 0 \leq i<j<|\Phi|\right\}
$$

For the example shown in Figure 2, there are a total of 12 letter recurrences in $\operatorname{REC}(\Phi)$, as shown in Table II.

\section{Previous Studies}

Our TRHA uses the temporal consistency constraints between the sets of recurring symbols to detect and localize the duplicate substrings. One way to do this is to apply brute-force pairwise matching to all letter recurrences in the recurrence set of the string $\Phi$ [15], as shown in Algorithm 1. Here, two recurrences $(i, j)$ and $(k, l)$ are assumed to belong to a pair of duplicate substrings if both positions of one recurrence are temporally closer than threshold $\delta$ to the corresponding positions of the other recurrence. The $\delta$ indicates the restricted
TABLE II

LETTER RECURRENCES DERIVED FROM STRING IN FIGURE 2 AND THEIR CORRESPONDING HASH VALUES. $\psi \in \hat{\Psi}$ : RECURRING LETTERS. $\operatorname{rec}(\psi) \in \operatorname{REC}(\Phi):$ RECURRENCE SET OF EACH $\psi \cdot\left\{\left(h_{1}, h_{2}\right)\right\}:$ SET OF RECURRENCE HASH VALUES OF LETTER RECURRENCES

\begin{tabular}{cll}
\hline$\psi$ & $\operatorname{rec}(\psi)$ & $\left\{\left(h_{1} ; h_{2}\right)\right\}$ \\
\hline 'W' & $\{(0,17)\}$ & $\{(0 ; 17)\}$ \\
'O' & $\{(1,9),(1,18),(9,18)\}$ & $\{(0 ; 8),(0 ; 17),(1 ; 9)\}$ \\
'R' & $\{(2,10),(2,19),(10,19)\}$ & $\{(0 ; 8),(0 ; 17),(2 ; 9)\}$ \\
'D' & $\{(3,20)\}$ & $\{(0 ; 17)\}$ \\
'S' & $\{(4,16),(4,21),(16,21)\}$ & $\{(0 ; 12),(0 ; 17),(3 ; 5)\}$ \\
'T' & $\{(7,12)\}$ & $\{(1 ; 5)\}$ \\
\hline
\end{tabular}

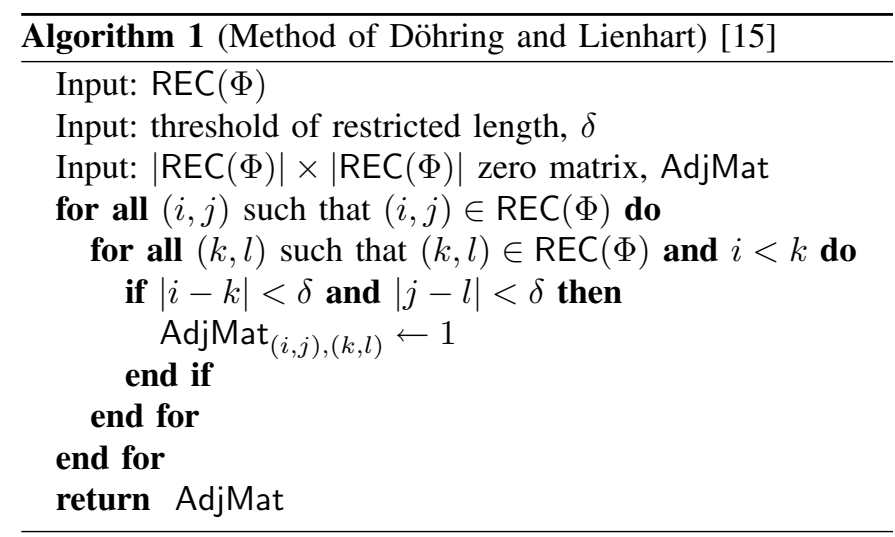

length of the duplicate substring. Output AdjMat is a twodimensional adjacency matrix, each element of which represents the matching result of each of two recurrences. Transitive closure is conducted on the basis of AdjMat to assemble recurring letters into duplicate substrings. Given $|\operatorname{REC}(\Phi)|$ denoting the number of letter recurrences, the computation cost in this case is $\left(\begin{array}{c}\operatorname{REC}(\Phi) \mid \\ 2\end{array}\right) \propto \Theta\left(|\operatorname{REC}(\Phi)|^{2}\right)$.

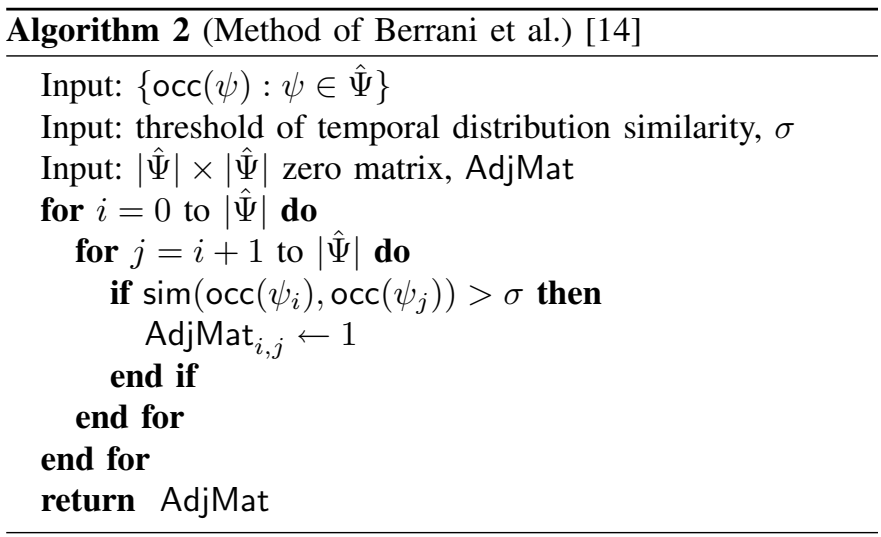

The computation cost can be reduced by applying bruteforce pairwise matching to the occurrence sets of all recurring letters in $\hat{\Psi}[14],[16]$ instead of to the letter recurrences. As 
shown in Algorithm 2, Berrani et al. [14] applied pairwise matching to all $\operatorname{occ}(\psi)$ sets so that two occurrence sets $\operatorname{occ}\left(\psi_{i}\right)$ and $\operatorname{occ}\left(\psi_{j}\right)$ with similar temporal distributions are assumed to belong to the same set of duplicate substrings. In Algorithm 2, $\operatorname{sim}\left(\operatorname{occ}\left(\psi_{i}\right), \operatorname{occ}\left(\psi_{j}\right)\right)$ indicates the similarity between the temporal distributions of two occurrence sets, the computation of which is similar to that in Algorithm 1. The $\sigma$ is the similarity threshold. Each element in AdjMat is a matching result for two occurrence sets. Putpuek et al. [16] used hierarchical clustering instead of pairwise matching for the same purpose. Given $|\hat{\Psi}|$ denoting the number of recurring symbols derived from string $\Phi$, the cost is $\left(\begin{array}{c}|\hat{\Psi}| \\ 2\end{array}\right) \propto \Theta\left(|\hat{\Psi}|^{2}\right)$.

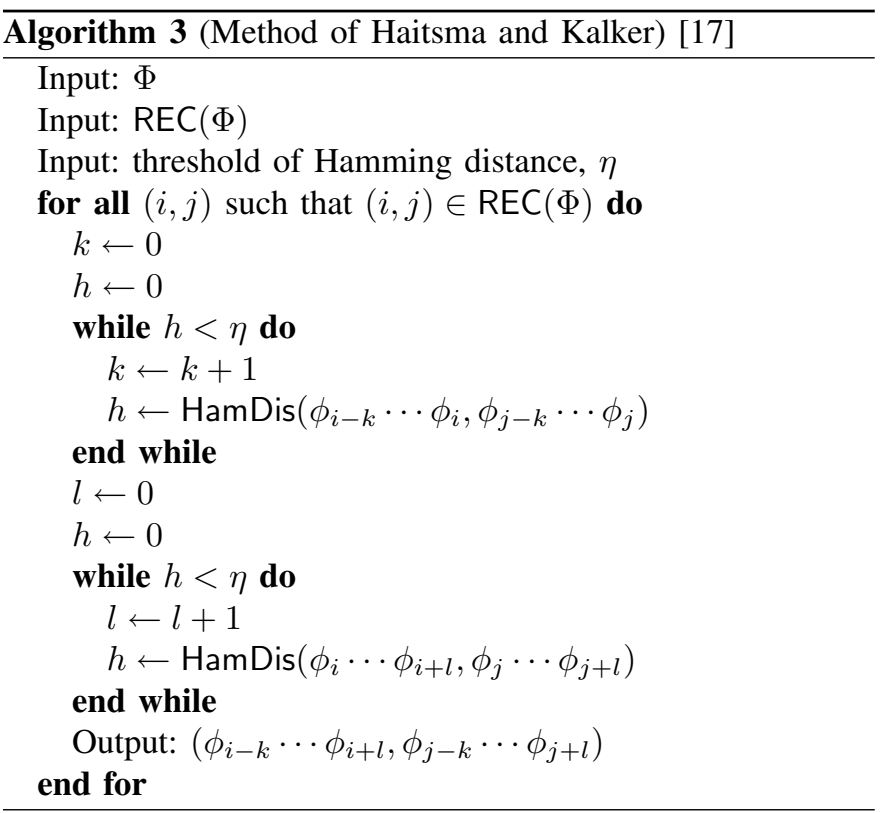

Haitsma and Kalker [17] proposed applying a letter growing strategy to all letter recurrences, the computation cost of which is $\Theta(|\operatorname{REC}(\Phi)|)$. As shown in Algorithm 3, once a letter recurrence $(i, j)$ is detected, the two letters $\phi_{i}$ and $\phi_{j}$ are combined with the preceding letters in string $\Phi$ so that two substrings are generated: $\phi_{i-1} \phi_{i}$ and $\phi_{j-1} \phi_{j}$. These two substrings are assumed to be duplicates if the Hamming distance HamDis() between them is less than a threshold $\eta$. This growing step is repeated in a backward and a forward manner until the Hamming distance is less than $\eta$. The number of operations required is $\Theta(|\operatorname{REC}(\Phi)|)$, which is much less than with the methods described above. However, the cost of a single operation in the growing process is much higher than that of other similarity measure processes, so the temporal alignment stage still requires lengthy processing.

In the string extraction field, the suffix tree method is considered to be a more classic method than others for the exact substring matching problem. A suffix tree is a tree structure the edges of which are labeled with substrings such that the paths from the root to the leaves have a one-to-one relationship with all possible suffixes of the entire string stream. Its use speeds up the operation of locating a substring in a stream. A suffix tree is effective for exact substring matching but not for approximate substring matching, especially for longer substrings. For instance, it cannot be used to formulate an entire video stream into an accurate fingerprint string due to decoding errors. In other words, even if a person identifies a repeated video sequence as an exact duplicate, the substrings formulated from this exact duplicate are never identical to each other. There are usually a number of letter mismatches between two duplicate substrings. Since a typical duplicate substring to be detected with our system is normally composed of around 100 or more letters, a suffix tree is not suitable for our exact-duplicate detection task due to the substantial length and the unavoidable mismatches of duplicate substrings.

\section{E. Temporal Recurrence Hashing}

We propose applying our second-stage hashing algorithm to all letter recurrences on the basis of their temporal alignment. The computational burden is $\Theta(|\operatorname{REC}(\Phi)|)$ with a much lower single-operation cost. For each letter recurrence $(i, j) \in \operatorname{REC}(\Phi)$, two recurrence hashing functions $h_{1}()$ and $h_{2}()$ are defined (Equations 5 and 6, where $r_{1}$ and $r_{2}$ are their resolution factors, respectively). The $h_{1}()$ corresponds to the temporal position of the first occurrence of the letter, and $h_{2}()$ corresponds to the temporal interval between the first and subsequent occurrences. These hashing functions should be robust enough so that multiple letter recurrences derived from two duplicate substrings have the same or very close hash value. For this reason, resolution factor $r_{1}$ should be set to no less than the restricted length of the duplicate substring to be detected.

In our experiments, because the duplicate video to be detected was a TV commercial, the duration of which was assumed to be no more than $30 \mathrm{~s}$, we set $r_{1}$ to 1 minute. On the other hand, for two letter recurrences derived from two duplicate substrings, the intra-recurrence intervals between the two positions of each recurrence should be the same or approximately identical to each other. The $h_{2}()$ is used to distinguish this type of symbol recurrence from irrelevant ones and noise. For the example in Figure 2, we set $r_{2}$ to 1 . However, due to decoding and fingerprinting errors, there are usually a few fragments differing between the intra-recurrence intervals of the fingerprint recurrences even if they are derived from duplicate videos. Therefore, the resolution factor of $h_{2}()$ was set to $1 \mathrm{~s}$ in our experiments so as to be robust against this problem. Table II lists the hash values of the symbol recurrences for $r_{1}=5$ and $r_{2}=1$.

$$
\begin{gathered}
h_{1}(i, j)=\text { floor }\left(\frac{i}{r_{1}}\right) \\
h_{2}(i, j)=\text { floor }\left(\frac{j-i}{r_{2}}\right)
\end{gathered}
$$

On the basis of $h_{1}()$ and $h_{2}()$, all letter recurrences are inserted into a two-dimensional hash table in which each dimension corresponds to a hash value. As shown in Figure 3, letter recurrences with high temporal consistency can be automatically assembled into the same bin or neighboring bins of the hash table. This second-stage hashing algorithm is summarized in Algorithm 4, from which we can see that the computational cost is $\Theta(|\operatorname{REC}(\Phi)|)$. Algorithm 4 is far more efficient than the algorithm of Döhring and Lienhart [15], the 


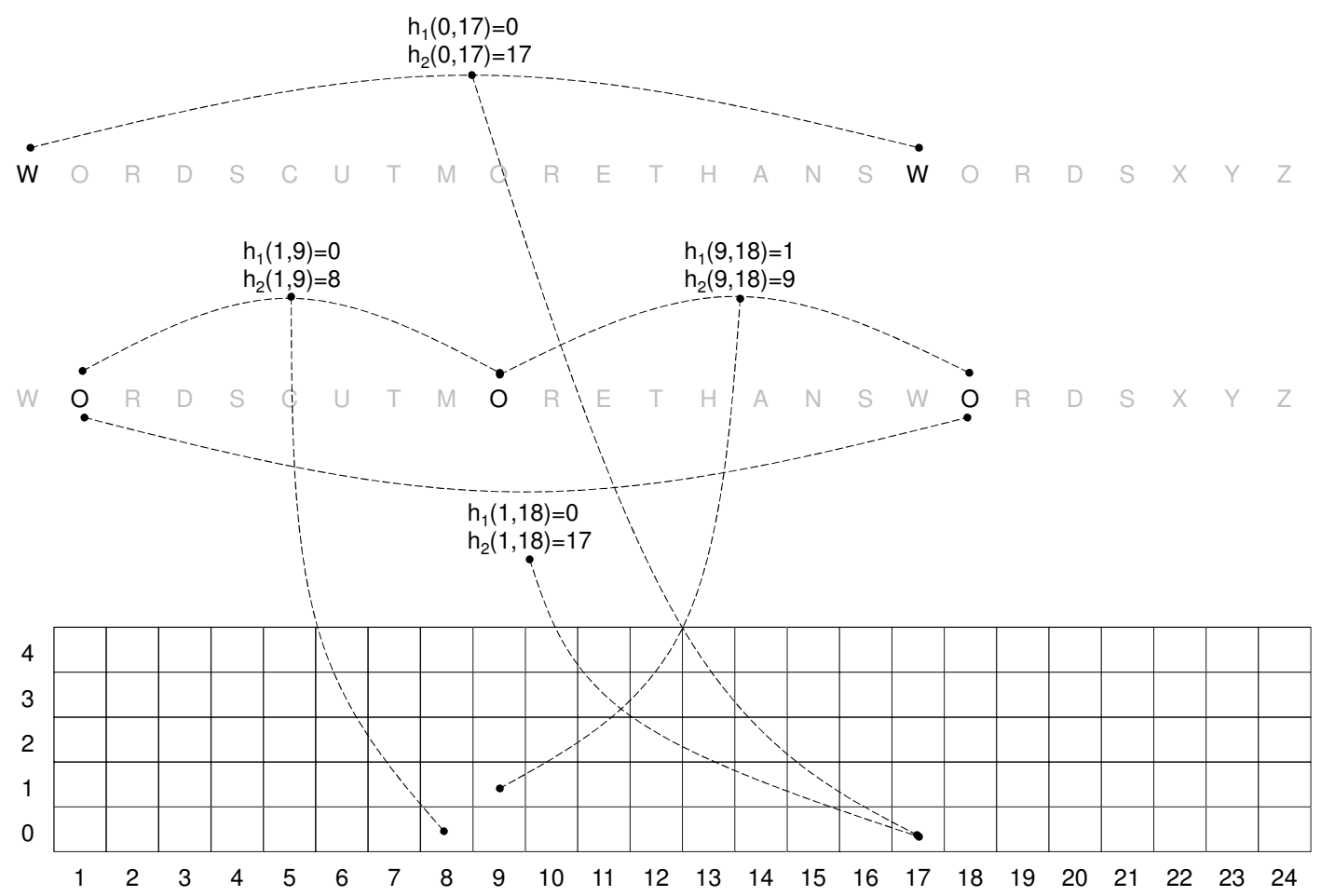

Fig. 3. Temporal recurrence hashing: $h_{1}(i, j)$ and $h_{2}(i, j)$ indicate recurrence hash values of symbol recurrence $(i, j)$. Lower table is two-dimensional hash table into which all letter recurrences are inserted on the basis of their recurrence hash values.

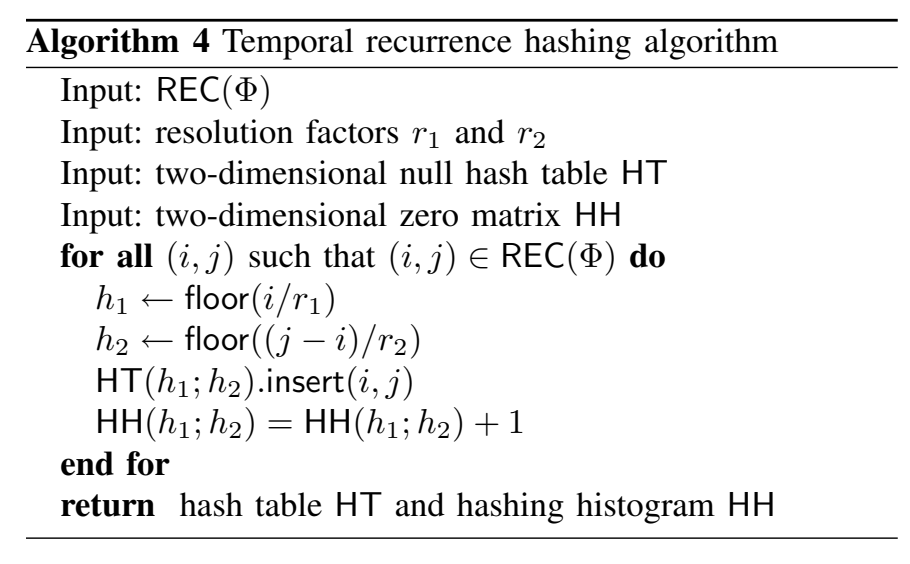

cost of which is $\Theta\left(|\operatorname{REC}(\Phi)|^{2}\right)$, because the former requires only linear time computations while the latter requires pairwise computations of temporal consistency. Algorithm 4 is also more efficient than that of Berrani et al. [14], the cost of which is $\Theta\left(|\hat{\Psi}|^{2}\right)$. For the example in Figure 2, the computational cost of Algorithm 4 is $\Theta(12)$ while that of the algorithm of Berrani et al. is $\Theta\left(\left(\begin{array}{l}6 \\ 2\end{array}\right)\right)=\Theta(15)$. Another example comes from our experiments: for a 1-month video, Algorithm 4 required 0.41 billion computations while that of Berrani et al. required 20 billion computations.

An example of a recurrence hash table is shown in Figure 5; it came from the string shown in Figure 2.

The recurring letters derived from duplicate substrings can be easily detected by searching for hash collisions in the twodimensional hash table. This is done by building a hashing histogram, as shown in Algorithm 4, concomitantly with the

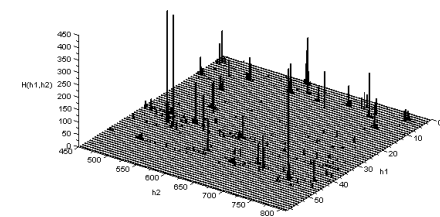

(a) Recurrence hashing histogram (b) Three local maximums

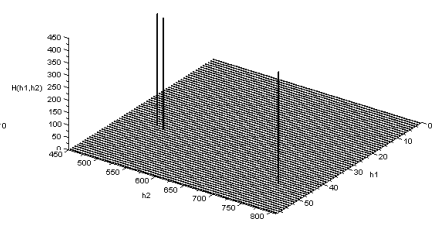

Fig. 4. Portion of recurrence hashing histogram of 1-hour video stream. Horizontal axes: recurrence hash values $\left(h_{1} ; h_{2}\right)$. Vertical axis: vote of letter recurrences.

construction of the hash table. Figure 4a shows an example hashing histogram built from a 1-hour video stream. For convenience, we show only a portion of it. The recurring letters derived from duplicate substrings are determined by detecting local maximums in the hashing histogram. We set a restricted duration threshold $\delta$, which is similar to that used in Algorithm 1, to filter out noise. Figure 4b shows the three local maximums detected from the hashing histogram shown in Figure 4a.

As described above, resolution factor $r_{1}$ should be large enough so that multiple letter recurrences derived from two duplicate substrings can have the same hash value. However, this condition cannot be satisfied in some cases. For the example in Figure 2, the two substrings $\phi_{9} \phi_{10}$ and $\phi_{18} \phi_{19}$ are duplicates, and the two letter recurrences derived from them are $(9,18)$ and $(10,19)$. If we set $r_{1}$ to 5 and $r_{2}$ to 1 , we get $h_{1}(9,18)=1, h_{1}(10,19)=2$, and $h_{2}(9,18)=h_{2}(10,19)=$ 9 . On the basis of these hash values, the two letter recurrences are inserted into two neighboring bins $(1 ; 9)$ and $(2 ; 9)$ instead of the same bin. To overcome this problem, when detecting 
local maximums from the two-dimensional hashing histogram, we replace each non-zero bin value with the average value of itself and its two closest neighbors in accordance with its $h_{1}()$ value.

The letter recurrences, the hash values of which correspond to a local maximum, are then assembled into pairs of duplicate substrings. For the example in Figure 2, we can detect three pairs of duplicate substrings: $\left(\phi_{1} \phi_{2}, \phi_{9} \phi_{10}\right)$ corresponding to local maximum $(0 ; 8),\left(\phi_{9} \phi_{10}, \phi_{18} \phi_{19}\right)$ corresponding to $(1 ; 9)$ and $(2 ; 9)$, and $\left(\phi_{0} \cdots \phi_{4}, \phi_{17} \cdots \phi_{21}\right)$ corresponding to local maximum $(0 ; 17)$. Figure 6 shows these three duplicate substring pairs.

\section{F. Post Processing}

To reduce the amount of memory usage, we construct the hashing histogram of letter recurrences without building a hash table. After detecting the local maximums, we recompute the two recurrence hashing values for each letter recurrence and assemble those, the hash values of which correspond to a local maximum. Since there are much fewer local maximums than bins in the histogram, memory usage is dramatically reduced. Due to errors introduced during the decoding and frame fingerprinting stage, there might also be holes or noise within the detected duplicate substrings. Morphological filtering is used to remove this clutter. The output is formatted as pairs of duplicate substrings. Transitive closure is used to combine these pairs into clusters. Similar to state-of-the-art works, we finally set a restricted duration threshold to remove noncommercial exact duplicates, e.g. television jingles etc.

The amount of computation for constructing the hashing histogram can be approximated as $|\operatorname{REC}(\Phi)|$, which is the number of letter recurrences derived from the string $\Phi$. The computation amount for detecting local maximums equals the number of non-zero bins in the hashing histogram, which is much less than $|\operatorname{REC}(\Phi)|$ so as to be negligible. The computation amount for assembling letter recurrences into duplicate substrings can also be approximated as $|\operatorname{REC}(\Phi)|$. Therefore, the maximal computation cost of the temporal recurrence hashing algorithm is $\Theta(|\operatorname{REC}(\Phi)|)$, which is much lower than those of related methods, e.g. $\Theta\left(|\operatorname{REC}(\Phi)|^{2}\right)[15]$ and $\Theta\left(|\hat{\Psi}|^{2}\right)$ [14], [16], described in Section III-D. We will further discuss the time complexity in Section V-A5.

\section{G. Multimedia Integration}

We demonstrate the effectiveness of applying the temporal recurrence hashing histogram to both video and audio sources in Section V-A4. Although the experimental results were already impressive, the performance was further improved by using an algorithm to integrate the exact-duplicate detectors for these two multimedia sources. Such integration can be at the feature, fingerprint, or decision level. For computational burden and memory usage reasons, we propose integration at the decision level. The observation that both video and audio duplicate detectors achieved almost $100 \%$ sequencelevel recall inspired us to use an intersection operation for integrating the detected exact-duplicate clips. This lowers the rate of false alarm detection with almost no increase in the number of misses. Another observation is that the framelevel recall is lower than the precision in both the video and audio cases. This inspired us to use a union operation for integrating the detected frames of each exact duplicate, which lowers the rate of misses without increasing the number of false alarms much. Section V-A4 demonstrates the feasibility of these assumptions.

\section{Cross-Stream Exact-Duplicate Cluster MATCHING AND GROUPING}

As mentioned in the introduction, we propose dividing large-scale video archives into multiple video streams of a certain length such that the computational effort and memory usage needed for processing each stream are low enough for practical use. For instance, in our experiments, a 5-year video archive was divided into 60 1-month video streams. Because there might be repetitions across the streams, the clusters of exact duplicates detected in all individual streams need to be matched and grouped together.

A straightforward way to do this is to concatenate all the exact-duplicate clusters into a very long stream and then apply the TRHA to this stream. However, such a stream would typically be much longer than the length that our proposed algorithm can handle due to memory requirements. Another way is to represent each exact-duplicate cluster with a key instance and to group these clusters by using instance-level fingerprint matching. However, the video or audio fingerprints derived from two instances of a given exact duplicate might be similar but not exactly the same due to decoding errors. The key instance matching strategy is usually not robust enough to this fingerprint difference, resulting in missed duplicate matches.

The individual-stream exact-duplicate detection, localization, and clustering algorithm does not have this problem because the transitive closure conducted in the post-processing step reduces the chance of misses. In the method proposed by Haitsma and Kalker [17], the Hamming distance, instead of strict matching like hashing, is used as the instance-level matching criterion to compensate for the fingerprint difference problem. However, this leads to false alarms when two commercials share similar visual layouts or background music. We propose using our BOFs model to solve this problem. Table III lists the notations used in this section.

TABLE III

EXPLANATION OF NOTATIONS USED IN SECTION IV.

\begin{tabular}{cl}
\hline Notation & Explanation \\
\hline$\tilde{\Psi}$ & $\begin{array}{l}\text { set of low-level fingerprints derived from a given exact- } \\
\text { duplicate cluster } \\
\text { low-level fingerprint }\end{array}$ \\
$\tau(\psi)$ & temporal code of a given fingerprint $\psi$ \\
BOF & bag of fingerprints descriptor of a given exact-duplicate \\
& cluster, which contains the temporal codes of all $\psi \in \tilde{\Psi}$ \\
$\tilde{\Psi}_{p, q}$ & set of mutual fingerprints shared between two clusters \\
$\operatorname{dis}()$ & distance between the BOF descriptors of two clusters \\
$\Delta(\psi)$ & $\begin{array}{l}\text { difference between the temporal codes for a given } \\
\text { mutual fingerprint } \psi \text { of two clusters }\end{array}$ \\
\hline
\end{tabular}




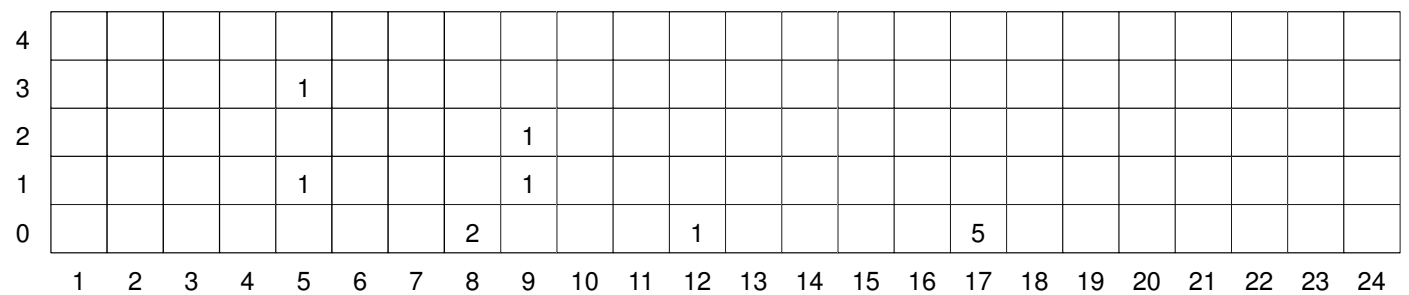

Fig. 5. Recurrence hash table. Number in each cell indicates number of letter recurrences inserted into corresponding bin.

\begin{tabular}{|c|c|c|c|c|c|c|c|c|c|c|c|c|c|c|c|c|c|c|c|c|c|c|c|c|}
\hline 0 & 1 & 2 & 3 & 4 & 5 & 6 & 7 & 8 & 9 & 10 & 11 & 12 & 13 & 14 & 15 & 16 & 17 & 18 & 19 & 20 & 21 & 22 & 23 & 24 \\
\hline W & 0 & $\mathrm{R}$ & D & S & C & $U$ & $\mathrm{~T}$ & $\mathrm{M}$ & 0 & $\mathrm{R}$ & $E$ & $\mathrm{~T}$ & $\mathrm{H}$ & $A$ & $\mathrm{~N}$ & S & W & 0 & $R$ & $D$ & S & $X$ & Y & Z \\
\hline W & 0 & $R$ & D & S & C & U & $\mathrm{T}$ & $M$ & 0 & $\mathrm{R}$ & $E$ & $\mathrm{~T}$ & $\mathrm{H}$ & $A$ & $N$ & S & W & $\mathrm{O}$ & $\mathrm{R}$ & D & S & $x$ & Y & Z \\
\hline W & 0 & $\mathrm{R}$ & $D$ & $S$ & C & $U$ & $T$ & $M$ & 0 & $\mathrm{R}$ & $E$ & $\mathrm{~T}$ & $\mathrm{H}$ & $A$ & $\mathrm{~N}$ & S & W & 0 & $\mathrm{R}$ & $D$ & $S$ & $X$ & Y & Z \\
\hline
\end{tabular}

Fig. 6. Three pairs of substrings identified as duplicates by detecting local maximums from recurrence hashing histogram.

\section{A. Bag-Of-Fingerprints Model}

In this model, each exact-duplicate cluster detected in the individual streams is regarded as a bag of fingerprints (BOFs) and is represented by their temporal distribution. First, the set of fingerprints derived from a cluster is denoted as $\tilde{\Psi}=\{\psi\}$ with $\psi$ indicating each fingerprint. Note that the fingerprints used here are those computed in the frame hashing stage, not the recurrence fingerprints discussed in Section III-E. Given a fingerprint $\psi \in \tilde{\Psi}$, we collect its relative positions within each instance of the cluster and compute the mean of these relative positions. We call this mean the 'temporal code of fingerprint $\psi$ ' and denote it as $\tau(\psi)$, which indicates the expected position at which fingerprint $\psi$ occurs in the duplicate video. The exact duplicate cluster can thus be represented as the complete set of temporal codes and is denoted as 'BOF $:=\{\tau(\psi): \psi \in \tilde{\Psi}\}$ '. This BOFs model is more robust against decoding errors than the key instance representation because it preserves consistency in the temporal orders of all local fingerprints. It also summarizes the temporal information of local fingerprints so as to be more compact than the clusterinstance representation.

\section{B. BOFs-Based Cluster Matching}

Given two clusters $\mathrm{BOF}_{p}$ and $\mathrm{BOF}_{q}$, the fingerprints shared by the clusters are called 'mutual fingerprints', and the set of mutual fingerprints is denoted as $\widetilde{\Psi}_{p, q}=\tilde{\Psi}_{p} \cap \tilde{\Psi}_{q}$. The distance between $\mathrm{BOF}_{p}$ and $\mathrm{BOF}_{q}$ is defined in Equation 7, where $\Delta(\psi)$ is the difference between the temporal codes of $\psi$ in the two clusters. The dis() shows how much variation or dispersion exists between the temporal orders of fingerprints derived from the two clusters. Two clusters are determined to be duplicates only if they share fingerprints with similar temporal alignments. In other words, even if two clusters share a large number of mutual fingerprints, the dis () might be larger if the mutual fingerprints are in inconsistent temporal order. This requirement reduces false alarms for local fingerprints. The duplicate clusters with a low dis() are thus grouped using transitive closure.

$$
\begin{aligned}
\operatorname{dis}\left(\mathrm{BOF}_{p}, \mathrm{BOF}_{q}\right) & =\sqrt{\frac{\sum_{\psi \in \tilde{\Psi}_{p, q}}(\Delta(\psi)-\bar{\Delta})^{2}}{\left|\tilde{\Psi}_{p, q}\right|}} \\
\Delta(\psi) & =\tau_{p}(\psi)-\tau_{q}(\psi) \\
\bar{\Delta} & =\frac{\sum_{\psi \in \tilde{\Psi}_{p, q}} \Delta(\psi)}{\left|\tilde{\Psi}_{p, q}\right|}
\end{aligned}
$$

\section{Evaluation}

\section{A. Individual-Stream Experiments}

\section{1) Data Preparation:}

We tested our exact-duplicate detection, localization, and clustering algorithm by using a 10-hour and a 1-month video stream. Both streams had been broadcast by a Japanese TV station. The 10-hour stream, with a manually annotated ground truth, was used for evaluating accuracy. The 1-month one was used for evaluating efficiency. We used a Linux server with an Intel Xeon 2.66-GHz CPU and 128 GB of main memory.

To the best of our knowledge, there are still no standard or state-of-the-art datasets for exact-duplicate detection. In TRECVID [26], a large dataset is provided for benchmarking near-duplicate detection tasks. We did not use this dataset because the TRECVID benchmark assumes that there are severe transformations between near duplicates. These transformations are non-existent in exact-duplicate videos, e.g. TV commercials. The fingerprinting strategies described in Section III-B are sensitive to these transformations, which is one of the limitations of our work. On the other hand, the query-based setting of the TRECVID benchmark differs greatly from that of our duplicate detection task in which neither query nor reference videos are provided beforehand. In particular, our duplicate detection task requires far more computational complexity than the TRECVID benchmark.

\section{2) Evaluation Criteria:}

In TRECVID [26], the mean average precision (MAP) is used for the evaluation of near-duplicate detection tasks. For each query, a list is created by ranking the similarity between each detection result and the query. The MAP is then 
computed on the basis of this list. However, this metric is not applicable to our duplicate detection task because our task is not query-based. Instead, it is more similar to a clustering task that groups video sequences into clusters so that the sequences in a group are duplicates of each other. We thus extended the classic purity metric, which is a standard external criterion of clustering quality, to our duplicate extraction task for evaluation.

We use $\mathbf{R}^{\mathbf{c}}=\left\{\mathbf{r}_{1}, \mathbf{r}_{2}, \cdots, \mathbf{r}_{\left|\mathbf{R}^{\mathbf{c}}\right|}\right\}$ to denote the results of exact-duplicate cluster detection and use $\mathbf{G}^{\mathbf{c}}=$ $\left\{\mathbf{g}_{1}, \mathbf{g}_{2}, \cdots, \mathbf{g}_{\left|\mathbf{G}^{\mathbf{c}}\right|}\right\}$ to denote the ground truth, where $\mathbf{r}$ and $\mathbf{g}$ each represent a TV commercial cluster. The sequence-level accuracy was evaluated by using precision $\mathcal{P}_{s}$ and recall $\mathcal{R}_{s}$ as defined in Equations 10 and 11 , where $\|\cdot\|$ indicates the number of video sequences in the TV commercial cluster. This is equivalent to assigning each result cluster $\mathbf{r}$ to ground-truth cluster $\mathbf{g}$, which has the highest ratio of overlapping to $\mathbf{r}$. The precision or recall of this assignment is then measured by counting the number of correctly assigned sequences and dividing it by the number of sequences in the result or groundtruth set. Only one result cluster can be assigned to one ground-truth cluster.

$$
\begin{aligned}
\mathcal{P}_{s} & =\frac{\sum_{i=1}^{\left|\mathbf{R}^{\mathbf{c}}\right|} \max _{j=1}^{\left|\mathbf{G}^{\mathbf{c}}\right|}\left\|\mathbf{r}_{i} \cap \mathbf{g}_{j}\right\|}{\sum_{i=1}^{\left|\mathbf{R}^{\mathbf{c}}\right|}\left\|\mathbf{r}_{i}\right\|} \\
\mathcal{R}_{s} & =\frac{\sum_{i=1}^{\left|\mathbf{R}^{\mathbf{c}}\right|} \max _{j=1}^{\left|\mathbf{G}^{\mathbf{c}}\right|}\left\|\mathbf{r}_{i} \cap \mathbf{g}_{j}\right\|}{\sum_{j=1}^{\left|\mathbf{G}^{\mathbf{c}}\right|}\left\|\mathbf{g}_{j}\right\|}
\end{aligned}
$$

Frame-level accuracy was defined for use in evaluating the exactness of localizing the beginning and end frames of an exact-duplicate video sequence. We focused on true positive sequences; i.e. false alarms and misses were discarded. The motivation is to make the evaluations of "detection" accuracy and "localization" accuracy independent on each other. Different from the sequence-level accuracy that takes false alarms and misses into consideration, the frame-level accuracy evaluates how exactly the algorithm can locate a commercial rather than whether the algorithm can detect it or not. $\mathbf{R}^{\mathbf{s}}=\left\{r_{1}, r_{2}, \cdots, r_{\left|\mathbf{R}^{\mathbf{s}}\right|}\right\}$ and $\mathbf{G}^{\mathbf{s}}=\left\{g_{1}, g_{2}, \cdots, g_{\left|\mathbf{G}^{\mathbf{s}}\right|}\right\}$ are used to respectively denote the detection results and the ground-truth set of true positive sequences with $\left|\mathbf{R}^{\mathbf{s}}\right|=\left|\mathbf{G}^{\mathbf{s}}\right|$, where $r$ and $g$ each represent a TV commercial instance. The precision and recall are defined as follows, where $\|\cdot\|$ indicates the number of frames.

$$
\begin{gathered}
\mathcal{P}_{f}=\frac{\sum_{i=1}^{\left|\mathbf{R}^{\mathbf{s}}\right|}\left\|r_{i} \cap g_{i}\right\|}{\sum_{i=1}^{\left|\mathbf{R}^{\mathbf{s}}\right|}\left\|r_{i}\right\|} \\
\mathcal{R}_{f}=\frac{\sum_{i=1}^{\left|\mathbf{G}^{\mathbf{s}}\right|}\left\|r_{i} \cap g_{i}\right\|}{\sum_{i=1}^{\left|\mathbf{G}^{\mathbf{s}}\right|}\left\|g_{i}\right\|}
\end{gathered}
$$

Note that a TV commercial for a product may be edited into versions of different lengths, e.g. 15- and 30-s versions. In this study, we regard these different versions as different commercials. Our proposed TRHA greatly depends on the consistency of temporal frame alignment between duplicate sequences. Therefore, it cannot detect two sequences as exact duplicates if their temporal alignments differ.

\section{3) Reference Techniques:}

We compared three state-of-the-art techniques, two videobased ones [14], [15] and one audio-based one [17]. Though these techniques were discussed in Section III-D from an algorithmic viewpoint, we discuss them here in more detail from the implementation viewpoint. Because the proposed TRHA is based on the repetition property of TV commercials, we compare it only with repetition-based techniques and do not consider knowledge-based techniques.

Near-duplicate detection techniques are also disregarded because the severe transformations assumed to exist between near duplicates are non-existent in exact duplicates. For instance, Poullot et al. [18], [19] described a feature indexing structure based on a Z-grid index and applied it to a querybased near-duplicate detection technique. This technique is robust against video transformation and efficient in local feature indexing. However, this efficiency is heavily dependent on a keyframe sampling strategy, and the indexing structure is not scalable enough to be applied to all the frames in a video stream. As a result, this technique cannot localize the exact beginning and end frames of duplicate sequences, which is a common limitation of most near-duplicate detection techniques.

Döhring and Lienhart [15] proposed using a frame hashing strategy based on a gradient histogram. A 1-s clip beginning from each frame is regarded as the basic unit of the video. This clip is to some extent similar to the fragment that is regarded as the basic unit in our proposed algorithm. Recurring clip pairs are detected in the frame hashing step. As mentioned in Section III-D, Döhring and Lienhart applied brute-force pairwise matching to all recurring clip pairs to assemble those pairs derived from recurring duplicate sequences into long and complete sequences. Post-processing methods, including noncommercial filtering based on restricted duration, sequence alignment, and transitive closure, are used to output the commercial clusters.

Berrani et al. [14] regarded keyframes extracted from shots as the basic unit of the video. These keyframes are clustered into a large number of small clusters on the basis of visual descriptors. This approach is an alternative to frame hashing. The small clusters are equivalent to the buckets in the hash table constructed during the frame hashing step. A pattern containing the temporal positions of cluster members is extracted from each small cluster; this pattern is similar to the occurrence set defined in Section III-C. The criterion for two temporal patterns to match is defined on the basis of the distribution of the temporal positions. Berrani et al. applied pairwise matching to all temporal patterns, as mentioned in Section III-D.

Haitsma and Kalker [17] described an exact-duplicate extraction algorithm based on audio frame hashing. Given two hash-identical fragments, two 3-second long blocks beginning from each fragment are regarded as the basic unit. The algorithm then iteratively merges these blocks with their neighboring fragments and evaluates their fingerprint dissimilarity on the basis of the Hamming distance. The iteration ends when the dissimilarity exceeds a threshold. This block-growing strategy is applied to all hash-identical fragment pairs. 
TABLE IV

EXPERIMENTAL RESULTS FOR INDIVIDUAL-STREAM EXACT-DUPLICATE DETECTION.

\begin{tabular}{cccccccc}
\hline & $\mathcal{P}_{s}(\%)$ & $\mathcal{R}_{s}(\%)$ & $\mathcal{F}_{s}(\%)$ & $\mathcal{P}_{f}(\%)$ & $\mathcal{R}_{f}(\%)$ & $\mathcal{F}_{f}(\%)$ & $\mathcal{T}(\mathrm{sec})$. \\
\hline TRHA-Video & $\mathbf{9 0 . 5 8}$ & $\mathbf{1 0 0}$ & $\mathbf{9 5 . 0 6}$ & $\mathbf{9 7 . 2 4}$ & $\mathbf{9 6 . 3 3}$ & $\mathbf{9 6 . 7 8}$ & $\mathbf{4}$ \\
Berrani [14] & 72.22 & 83.66 & 77.52 & 99.18 & 83.47 & 90.65 & 40 \\
Döhring [15] & 74.42 & 95.05 & 83.48 & 98.19 & 94.40 & 96.26 & 108 \\
\hline TRHA-Audio & $\mathbf{9 3 . 9 3}$ & $\mathbf{9 9 . 5 0}$ & $\mathbf{9 6 . 6 3}$ & $\mathbf{9 9 . 4 3}$ & $\mathbf{9 2 . 0 3}$ & $\mathbf{9 5 . 5 9}$ & $\mathbf{6}$ \\
Haitsma [17] & 82.16 & 98.02 & 89.39 & 97.89 & 86.69 & 91.95 & 40 \\
\hline TRHA-Integration & $\mathbf{9 6 . 6 3}$ & $\mathbf{9 9 . 5 0}$ & $\mathbf{9 8 . 0 5}$ & $\mathbf{9 7 . 3 4}$ & $\mathbf{9 7 . 4 4}$ & $\mathbf{9 7 . 3 9}$ & $\mathbf{1 0}$ \\
\hline
\end{tabular}

\section{4) Experimental Results:}

Table IV summarizes the performance results for individualstream exact-duplicate extraction. 'TRHA-Video' and 'TRHAAudio' represent the results of applying the TRHA to an individual source, while TRHA-Integration represents the results of multimedia integration. A 10 -hour video stream was used. The $\mathcal{F}_{s}$ and $\mathcal{F}_{f}$ represent the $\mathrm{F} 1$ score, and $\mathcal{T}$ represents the processing time. Note that $\mathcal{T}$ does not include video decoding and fingerprint computation, but only the pure exact-duplicate detection and localization. We did not compare the runtime of the former part because it is consistent between our algorithm and all three baselines.

We can see that both TRHA-Video and TRHA-Audio outperformed the baselines for all three criteria. The TRHA detects recurring fragments by searching for hash-identical fragments, while the algorithms of Döhring and Lienhart and of Haitsma and Kalker search for hash-similar clips on the basis of respectively the Euclidean distance and the Hamming distance. The latter strategy led to more false-alarm duplicate detection, which explains the lower sequence-level precision of the two baselines. We also found that the clusteringbased solution of frame hashing used in the algorithm of Berrani et al. showed both lower discriminative power and lower robustness than the fingerprinting-based solutions used in the other algorithms. This explains the lower sequencelevel accuracy of their baseline. Their method uses a keyframe instead of a frame or fragment as the basic unit of the video stream, so the exact boundaries of exact duplicates are difficult to precisely detect, which led to the lower frame-level recall. The lower recall of the baseline of Haitsma and Kalker is thought to be due to the use of the block growing strategy based on the Hamming distance.

All the false alarms with TRHA-Video and TRHA-Audio were non-commercial duplicates including program jingles and highlights and rebroadcast news sequences. Some of them were duplicates in both the video and audio streams, while others were duplicates in either one or the other. By integrating the video and audio exact-duplicate matches, the false alarms of the latter type were successfully removed, and $\mathcal{F}_{s}$ was improved to $98.1 \%$. In addition, the frame-level information loss introduced by the framing error was moderated, and $\mathcal{F}_{f}$ was improved to $97.4 \%$.

Given $|\operatorname{REC}(\Phi)|$ denoting the number of recurring fragment pairs derived from the stream, the complexity of Döhring and Lienhart's algorithm is $\left({ }_{2}^{|\operatorname{REC}(\Phi)|}\right)$. Given $|\hat{\Psi}|$ denoting the number of recurring fingerprints derived from the stream, the complexity of the algorithm of Berrani et al. is $\left(\begin{array}{c}|\hat{\Psi}| \\ 2\end{array}\right)$. As introduced in Section III-D, the complexity of our proposed detection algorithm and Haitsma and Kalker's algorithm is $|\operatorname{REC}(\Phi)|$. In most cases, $\left(\begin{array}{c}\operatorname{REC}(\Phi) \mid \\ 2\end{array}\right)>\left(\begin{array}{c}|\hat{\mid}| \\ 2\end{array}\right)>|\operatorname{REC}(\Phi)|$, which can be confirmed from the processing times listed in Table IV and the video data statistics listed in Table V. Haitsma and Kalker's baseline did not show higher efficiency than that of Berrani et al. because of the high operation cost of the iterative block growing strategy it uses.

Note that if $|\operatorname{REC}(\Phi)|>\left(\begin{array}{c}|\hat{\Psi}| \\ 2\end{array}\right)$, the complexity of our algorithm becomes higher than that of Berrani's baseline. An extreme example is a long video containing only one commercial that is repeatedly broadcast a large number of times. In this case, $\left(\begin{array}{c}|\hat{\Psi}| \\ 2\end{array}\right)$ is small because of the small number of commercial, while $|\operatorname{REC}(\Phi)|$ is large because of the high frequency of the same commercial. In other words, our algorithm might require higher complexity than Berrani's baseline if the video sequence contains only a small number of different exact duplicates, each of which derives a very large number of recurrences. However, this isolated case fairly infrequently happens in the real world. We tested 601 month video sequences in our experiments, and in all cases, $|\operatorname{REC}(\Phi)| \ll\left(\begin{array}{c}|\hat{\Psi}| \\ 2\end{array}\right)$, i.e. our algorithm would require much lower complexity than Berrani's baseline.

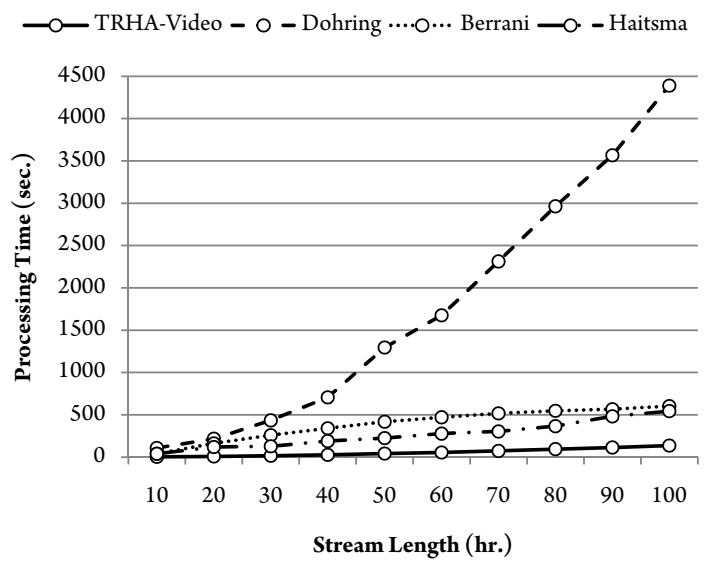

Fig. 7. Relationship between computation complexity and stream length.

Another experiment was conducted to investigate the relationship between the computation complexity and the stream length. Video streams with different lengths were extracted from a 1-month stream. As shown in Figure 7, the processing time increased almost linearly with the stream length with the TRHA and the baselines of Berrani et al. and Haitsma and Kalker. In contrast, the curve for the baseline of Döhring and 
Lienhart shows a more than proportional increase in processing time with the stream length. The much shorter processing time with TRHA demonstrates that it has a higher level of efficiency than the other techniques. We further investigated its efficiency by applying it to a 1-month video stream. The processing time was 50 minutes for video and 42 minutes for audio. This confirmed its high level of efficiency. Future work includes comparing the efficiency of our detection algorithm with that of other techniques using the same 1-month video stream.

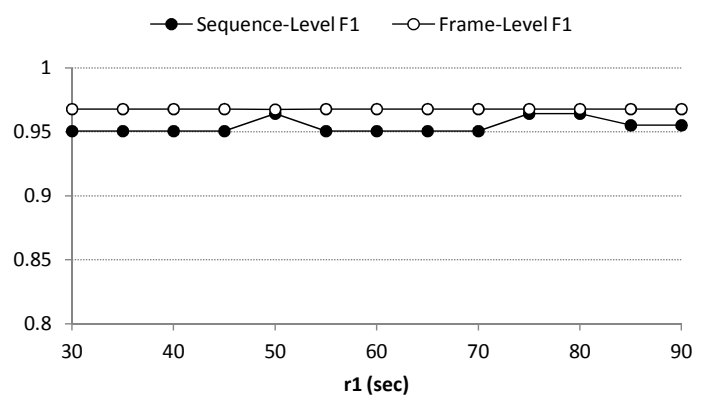

(a) Sensitivity to $r_{1}$. (video case, $r_{2}=1 \mathrm{sec}$.)

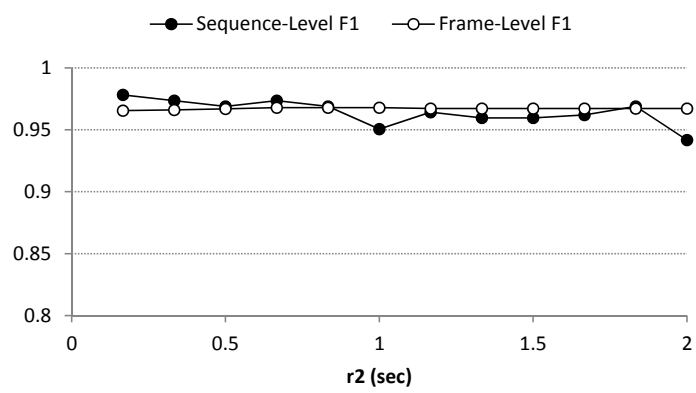

(b) Sensitivity to $r_{2}$. (video case, $r_{1}=60 \mathrm{sec}$.)

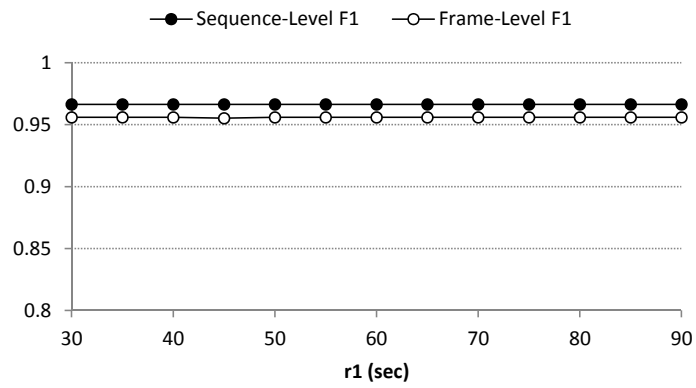

(c) Sensitivity to $r_{1}$. (audio case, $r_{2}=1 \mathrm{sec}$.)

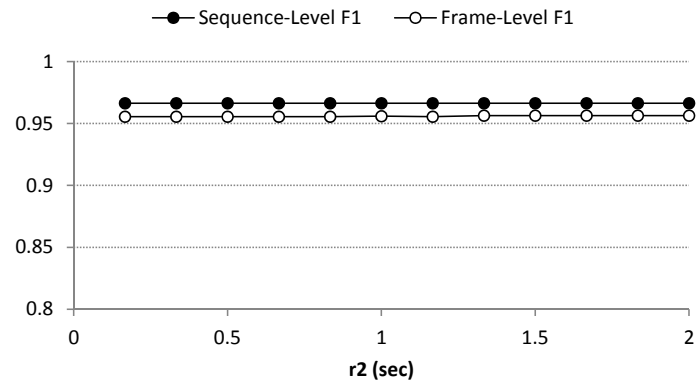

(d) Sensitivity to $r_{2}$. (audio case, $r_{1}=60 \mathrm{sec}$.)

Fig. 8. Sensitivity of TRHA to both parameters $r_{1}$ and $r_{2}$.
We further investigate the sensitivity of our algorithm to the two resolution factors $r_{1}$ and $r_{2}$, which is shown in Figure 8. The detection and localization accuracies were evaluated by fixing one parameter and varying the other. From these graphs, we can see that our performance is consistent between both parameters. The runtime for each individual parameter setting was almost the same as $\mathcal{T}$ listed in Table IV. However, it should be noted that a higher resolution (lower $r_{1}$ and $r_{2}$ values) enlarges the size of the recurrence hash table and thus increases the memory usage. In our experiments, we set $r_{1}$ to 1 minute and $r_{2}$ to 1 second for evaluation.

5) Further Discussion of Time Complexity:

As described in Section V-A4, we applied our TRHA to a 1month video stream. The processing time was 50 minutes for video and 42 minutes for audio. The statistics listed in Table $\mathrm{V}$ provide more insight into the time complexity of the proposed algorithm. We follow the notations used in Section III and explained in Table I: $n_{f}$ indicates the number of frames; $|\Phi|$ the number of letters, i.e. the length of the string formulated from the 1-month stream; $|\Psi|$ the number of letters occurring in the string; $|\hat{\Psi}|$ the number of recurring letters occurring at least twice; $|\operatorname{REC}(\Phi)|$ the number of all letter recurrences; $n_{b}$ the number of non-zero bins in the recurrence hashing histogram; and $n_{m}$ the number of local maximums detected from the hashing histogram.

TABLE V

STATISTICS ON TIME COMPLEXITY OF TRHA APPLIED TO 1-MONTH VIDEO STREAM. UNIT: MILLIONS.

\begin{tabular}{lrr}
\hline Statistic & Video & Audio \\
\hline$n_{f}$ & 77.8 & 77.8 \\
$|\Phi|$ & 19.0 & 74.0 \\
$|\Psi|$ & 7.4 & 59.8 \\
$|\hat{\Psi}|$ & 0.2 & 1.4 \\
$|\operatorname{REC}(\Phi)|$ & 411.9 & 78.9 \\
$n_{b}$ & 201.6 & 17.9 \\
$n_{m}$ & 0.3 & 0.4 \\
\hline
\end{tabular}

An interesting observation here is that the video and audio streams showed large variation in certain statistics though the mining of exact duplicates was similar in terms of effectiveness and efficiency for the two streams. The reason lies in the different characteristics of the two media formats and their corresponding fingerprinting strategies. A video stream is usually comprised of sets of continuous frames that are visually similar to each other, while an audio stream is comprised of more discrete frames that are acoustically different from each other. If we define the combination of continuous frames with the same fingerprint as a fragment, then the length of a video fragment will be longer than that of an audio one in most cases. As a result, the number of video fragments will be smaller than that of audio ones because the video and audio streams have the same length. This explains the lower $|\Phi|$ value in the video case listed in Table V. On the other hand, the video fingerprint provided lower discriminative power than the audio one in capturing the low-level features of each fragment. As a result, the algorithm detected noisier letter recurrences in the video stream than the audio one. This explains the higher $|\operatorname{REC}(\Phi)|$ value in Table $\mathrm{V}$ for the video stream. 
The differences in the statistics listed in Table $\mathrm{V}$ result in different processing times for exact-duplicate detection. Table VI lists the processing times for the four main steps in our detection algorithm. Here, we discuss the relationship between the statistics listed in Table $\mathrm{V}$ and the processing times listed in Table VI to provide an insight into the time complexity of our algorithm.

TABLE VI

PROCESSING TIME (MM:SS) OF DETECTING EXACT DUPLICATES IN 1-MONTH VIDEO STREAM.

\begin{tabular}{lll}
\hline Step & Video & Audio \\
\hline Frame Hashing & $03: 07$ & $07: 29$ \\
Temporal Recurrence Hashing & $09: 39$ & $02: 36$ \\
Local Maximum Detection & $12: 22$ & $01: 03$ \\
Symbol Recurrence Assembling & $23: 21$ & $29: 13$ \\
\hline
\end{tabular}

The frame hashing step discussed here includes only two processes: 1) combining continuous fingerprint-identical frames into a fragment and 2) constructing a one-dimensional hash table. The video decoding and fingerprint computation processes are not considered. Let $c_{1}$ denote the single operation cost of processing each frame and $c_{2}$ that of inserting each fragment (each letter) into the hash table. The cost of frame hashing can be approximated by $\Theta\left(n_{f} c_{1}+|\Phi| c_{2}\right)$. Since $c_{1}$ is smaller than $c_{2}$, the ratio of the processing time between the audio and video streams is theoretically within the range $\left[\left(n_{f}^{a}+|\Phi|^{a}\right) /\left(n_{f}^{v}+|\Phi|^{v}\right),|\Phi|^{a} /|\Phi|^{v}\right] \equiv[1.57,3.88]$. From Table VI and with a bit of calculation, we can see that this ratio equaled 2.40 .

Let $c_{3}$ denote the single operation cost of processing each fragment (each letter) and $c_{4}$ that of voting each letter recurrence into the hashing histogram. The cost of temporal recurrence hashing can be approximated by $\Theta\left(|\Phi| c_{3}+|\operatorname{REC}(\Phi)| c_{4}\right)$. Since $c_{3}$ is smaller than $c_{4}$, the ratio of the processing time between the audio and video streams is theoretically within the range $\left[|\operatorname{REC}(\Phi)|^{a} /|\operatorname{REC}(\Phi)|^{v},\left(|\Phi|^{a}+|\operatorname{REC}(\Phi)|^{a}\right) /\left(|\Phi|^{v}+\right.\right.$ $\left.\left.|\operatorname{REC}(\Phi)|^{v}\right)\right] \equiv[0.19,0.35]$. From Table VI and with a bit of calculation, we can see that this ratio equaled 0.27.

Let $c_{5}$ denote the single operation cost of local maximum discrimination. The cost of local maximum detection can be approximated by $\Theta\left(n_{b} c_{5}\right)$. The ratio of the processing time between the audio and video streams is theoretically $n_{b}^{a} / n_{b}^{v}=$ 0.089. From Table VI and with a bit of calculation, we can see that this ratio equaled 0.085 .

Let $c_{6}$ denote the single operation cost of processing each fragment (each letter), $c_{7}$ that of re-computing the recurrence fingerprints for each letter recurrence, and $c_{8}$ that of assembling the letter recurrences in each local maximum bin. The cost of letter recurrence assembling can be approximated by $\Theta\left(|\Phi| c_{6}+|\operatorname{REC}(\Phi)| c_{7}+n_{m} c_{8}\right)$. Since $c_{6}$ and $c_{7}$ are much smaller than $c_{8}$, the cost can be further approximated by $\Theta\left(n_{m} c_{8}\right)$. The ratio of the processing time between the audio and video streams is theoretically $n_{m}^{a} / n_{m}^{v}=1.26$. From Table VI and with a bit of calculation, we can see that this ratio equaled 1.25 .

In summary, the maximum computation cost of the core steps is $\Theta(|\operatorname{REC}(\Phi)|)$, which is much lower than that of
Döhring and Lienhart's method $|\operatorname{REC}(\Phi)|^{2}$ and that of Berrani et al. $|\hat{\Psi}|^{2}$. The most time-consuming step was letter recurrence assembling (Table VI) because of the large operational cost of $c_{8}$. Future work includes refining the implementation of this step to further improve efficiency. Note that the computational cost of letter recurrence assembling is constant over our algorithm and over those of Döhring and Lienhart and of Berrani et al.

\section{B. Cross-Stream Experiments}

We tested our cross-stream exact-duplicate cluster matching and grouping algorithm based on the bag-of-fingerprints (BOFs) model by using a 5-month video database containing five 1-month streams respectively broadcast from five different channels. We chose six hours out of each 1-month stream and annotated them to create a 30-hour ground-truth set. This set contained $608 \mathrm{TV}$ commercials and $1150 \mathrm{TV}$ commercial instances. We individually applied the TRHA to the 1-month streams in parallel. To evaluate the performance of crossstream exact-duplicate cluster matching and grouping, we focused on the clusters containing TV commercials broadcast across multiple channels. This subset contained 229 commercials and 757 instances.

We defined under-grouping and over-grouping error rates as respectively shown in Equations 14 and 15. Under-grouping represents the case in which the algorithm failed to detect two duplicates as identical, so a ground-truth exact-duplicate group was separated into multiple ones. In contrast, overgrouping represents the case in which the algorithm failed to differentiate two non-duplicate clusters, so multiple groundtruth exact-duplicate groups were merged into one group. A perfect algorithm would avoid both cases, and the two error rates defined in Equations 14 and 15 would be zero. $\nu_{u}$ and $\nu_{o}$ are respectively the number of under-grouped and overgrouped exact duplicate groups. The $\nu_{\mathrm{g}}=229$ is the total number of exact-duplicate groups in the ground-truth set. We compared the results of our match and group algorithm with the baseline implemented on the basis of the Hamming distance [17]. This baseline represents each exact-duplicate cluster by using a key instance and uses the audio fingerprint as the feature descriptor. Two clusters are determined to be identical if the Hamming distance between the fingerprints of the two key instances exceeds a certain threshold. The experimental results for cross-stream exact-duplicate cluster matching and grouping are listed in Table VII.

$$
\begin{gathered}
\rho_{u}=\frac{\nu_{u}}{\nu_{\mathrm{g}}} \\
\rho_{o}=\frac{\nu_{o}}{\nu_{\mathrm{g}}}
\end{gathered}
$$

TABLE VII

EXPERIMENTAL RESULTS FOR CROSS-STREAM EXACT-DUPLICATE CLUSTER MATCHING AND GROUPING.

\begin{tabular}{cccccc}
\hline & $\nu_{u}$ & $\rho_{u}(\%)$ & $\nu_{o}$ & $\rho_{o}(\%)$ & $\mathcal{T}$ (min.) \\
\hline BOFs & $\mathbf{3}$ & $\mathbf{1 . 3 1}$ & $\mathbf{1 0}$ & $\mathbf{4 . 3 7}$ & 42 \\
Haitsma [17] & 14 & 6.11 & 10 & 4.37 & 48 \\
\hline
\end{tabular}


As mentioned above, a straightforward approach to this task is to concatenate all detected exact-duplicate clusters into a very long video stream and then apply the TRHA to this stream. We did not take this approach because the concatenated stream would be too long for the TRHA to handle due to the large memory usage. Another approach, as mentioned above, is to represent each exact-duplicate cluster with a key instance and to group the clusters on the basis of instance-level fingerprint matching. The baseline implemented on the basis of the Hamming distance corresponds to this approach. From Table VII, we can see that our algorithm output fewer under-grouped groups than the baseline.

The main reason for an under-grouping lies in the sensitivity of the feature descriptors to noises. For instance, due to decoding errors, the video or audio features derived from two instances of a given exact-duplicate cluster might differ so that the algorithm failed to detect the two duplicates as identical. The baseline, which represents each exact-duplicate cluster with only a single key instance, is especially sensitive to this kind of noise. In contrast, the BOFs model used in our algorithm preserves the temporal feature of the local fingerprints derived from all instances instead of one single key instance, so the matching process is more robust against decoding errors than the baseline. The three under-grouping cases were not discarded by our algorithm due to the small number of instances in the detected exact-duplicate clusters.

The main reason for an over-grouping lies in the low discriminative power of the feature descriptor or the matching criterion. From Table VII, we can see that the over-grouping error rate of our algorithm was the same as that of the baseline. The BOFs-based cluster matching criterion discovers false matches of local fingerprints by investigating their temporal alignment and does not increase the chance of over-grouping compared to the baseline.

\section{Application to TV Commercial Mining}

A promising application of our system is TV commercial mining in which the chronological distribution of the massive number of TV commercials is visualized, enabling rediscovery of knowledge related to advertising. An example visualization of information related to TV commercials extracted from a one-year and five-channel broadcast video archive is illustrated in Figure 9. There are nine types of chronological distributions involving five types of information: broadcast frequency, date, hour of the day, day of the week, and channel.

The visualization of information for a Japanese beer commercial is illustrated in Figure 10. Figure 10b shows that this commercial was most frequently broadcast after $6 \mathrm{pm}$, which makes sense because Japanese white collar workers normally finish work around $6 \mathrm{pm}$. The blank period between 2 am and 11 am also makes sense as there is a self-imposed restriction in Japan that forbids the broadcasting of alcoholrelated TV commercials between 2 am and 11 am on weekdays and between 2 am and $5 \mathrm{pm}$ on weekends. The distribution across days and during the day was fairly smooth, as shown in Figure 10c. The visualizations for other alcohol-related TV commercials showed similar characteristics.
The visualization of information for a car commercial is illustrated in Figure 11. There are three periods with higher broadcast frequency, as shown in $11 \mathrm{~b}$. The first one is from 6 am to 8 am, when most white collar workers are having breakfast and probably watching TV. The second one is around $12 \mathrm{am}$, when most of them are having lunch and may again be watching TV. The third one is after $6 \mathrm{pm}$, when most of them have finished work. The knowledge that can be rediscovered from this is that this car was targeted at white collar workers. Such rediscovered knowledge, which we make more accessible, can be transformed into business intelligence for producers, distributors, and advertisers.

\section{CONCLUSION}

Our proposed system based on exact-duplicate matching detects and localizes TV commercials in a video stream, clusters the exact duplicates, and detects duplicate exact-duplicate clusters across video streams. The two-stage temporal recurrence hashing algorithm used for the detection, localization, and clustering is fully unsupervised, generic, and ultrahigh speed. Its performance is consistent between video and audio streams. In a test, it detected and localized the exact-duplicate TV commercials from a 1-month video streams in less than 42 minutes. The algorithm used to integrate the video and audio streams improves extraction performance. Its sequenceand frame-level accuracies in testing were respectively $98.1 \%$ and $97.4 \%$. The algorithm used to detect duplicate exactduplicate clusters across multiple streams is based on a new bag-of-fingerprints model. It is robust against decoding errors. Testing using 10-hour, 1-month, and 5-year video streams demonstrated the effectiveness and efficiency of this system.

We are currently investigating the feasibility of fingerprintlevel integration of video and audio duplicate extraction strategies. We plan to investigate the application of this system to other fingerprinting strategies to determine whether it is invariant to different fingerprinting techniques and plan to extend its application to a three-dimensional distribution visualization strategy to further improve the knowledge discovery capability.

\section{REFERENCES}

[1] J. Wang, L. Duan, L. Xu, H. Lu, and J. S. Jin, "Tv ad video categorization with probabilistic latent concept learning," in Proceedings of the international workshop on Workshop on multimedia information retrieval, 2007, pp. 217-226.

[2] R. Lienhart, C. Kuhmunch, and W. Effelsberg, "On the detection and recognition of television commercials," in IEEE International Conference on Multimedia Computing and Systems '97, Jun. 1997, pp. 509516.

[3] A. Hauptmann and M. Witbrock, "Story segmentation and detection of commercials in broadcast news video," in IEEE International Forum on Research and Technology Advances in Digital Libraries, Apr. 1998, pp. $168-179$.

[4] A. Albiol, M. Ch, F. Albiol, and L. Torres, "Detection of tv commercials," in IEEE International Conference on Acoustics, Speech, and Signal Processing, vol. 3, May 2004, pp. 541-544.

[5] X.-S. Hua, L. Lu, and H.-J. Zhang, "Robust learning-based tv commercial detection," in IEEE International Conference on Multimedia and Expo, Jul. 2005, pp. 149-152.

[6] L.-Y. Duan, J. Wang, Y. Zheng, J. S. Jin, H. Lu, and C. Xu, "Segmentation, categorization, and identification of commercial clips from tv streams using multimodal analysis," in Proceedings of the 14th annual ACM international conference on Multimedia, 2006, pp. 201-210. 

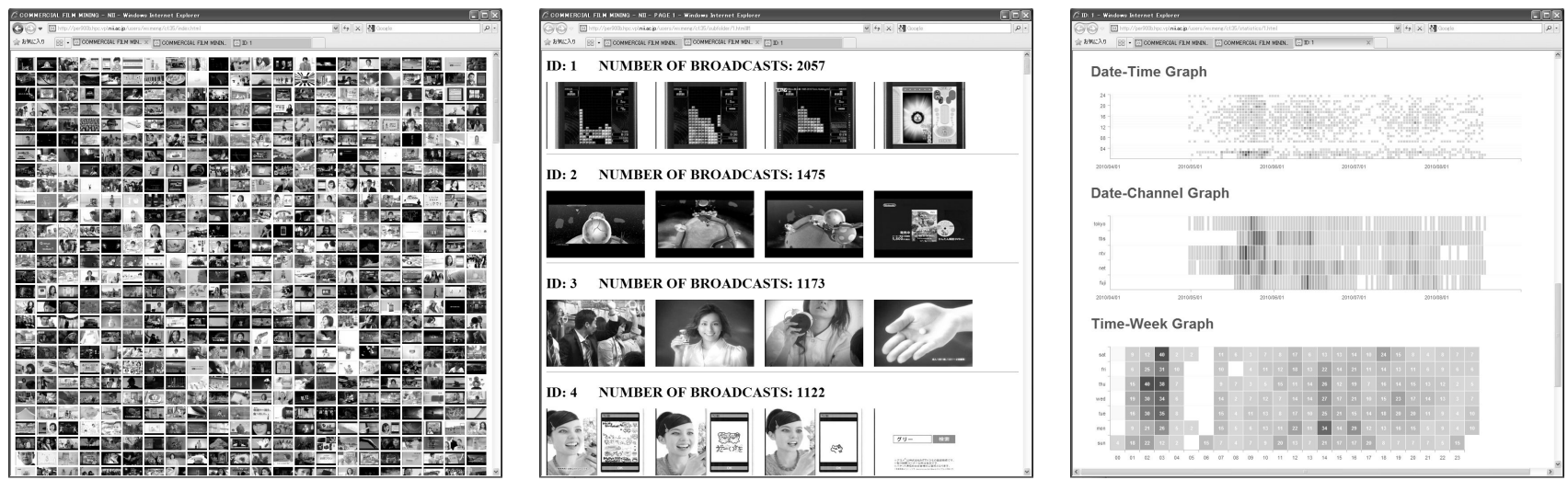

Fig. 9. Visualization of information related to extracted TV commercials.

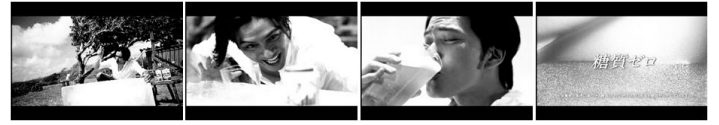

(a) Keyframes.

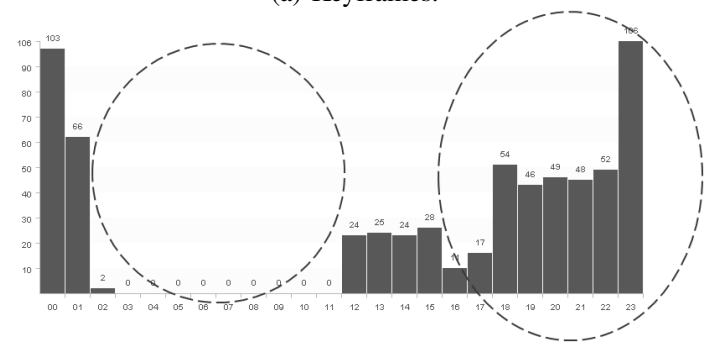

(b) Horizontal axis: hour of the day. Vertical axis: broadcast frequency.

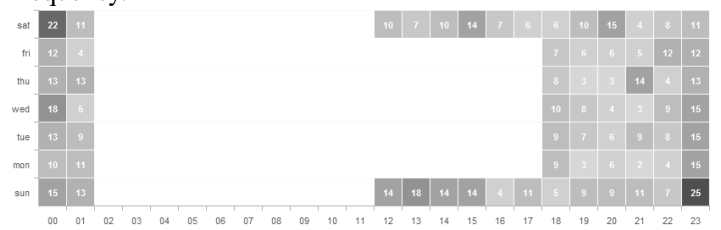

(c) Horizontal axis: hour of the day. Vertical axis: day of the week. Darker blocks indicate time zones with higher broadcast frequency.

Fig. 10. Visualization of information related to Asahi Breweries TV commercial.

[7] Y. Li, D. Zhang, X. Zhou, and J. S. Jin, "A confidence based recognition system for tv commercial extraction," in Proceedings of the nineteenth conference on Australasian database, 2008, pp. 57-64.

[8] J. Gauch and A. Shivadas, "Identification of new commercials using repeated video sequence detection," in IEEE International Conference on Image Processing, vol. 3, Sep. 2005, pp. 1252-1255.

[9] Y. Li, J. Jin, and X. Zhou, "Matching commercial clips from tv streams using a unique, robust and compact signature," in Digital Image Computing: Technqiues and Applications, Dec. 2005, pp. 266-272.

[10] M. Covell, S. Baluja, and M. Fink, "Advertisement detection and replacement using acoustic and visual repetition," in 2006 IEEE 8th Workshop on Multimedia Signal Processing, Oct. 2006, pp. 461-466.

[11] J. M. Gauch and A. Shivadas, "Finding and identifying unknown commercials using repeated video sequence detection," Comput. Vis. Image Underst., vol. 103, no. 1, pp. 80-88, July 2006.

[12] C. Herley, "Argos: automatically extracting repeating objects from multimedia streams," IEEE Transactions on Multimedia, vol. 8, no. 1, pp. 115-129, Feb. 2006.

[13] A. Shivadas and J. Gauch, "Real-time commercial recognition using color moments and hashing," in Fourth Canadian Conference on Computer and Robot Vision, May 2007, pp. 465-472.

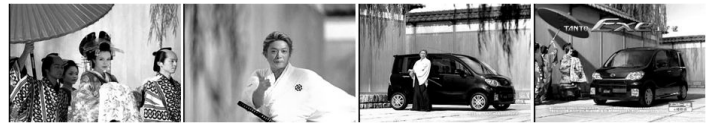

(a) Keyframes.

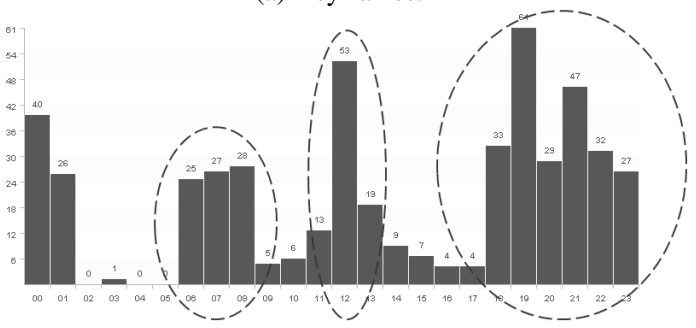

(b) Horizontal axis: hour of the day. Vertical axis: broadcast frequency.

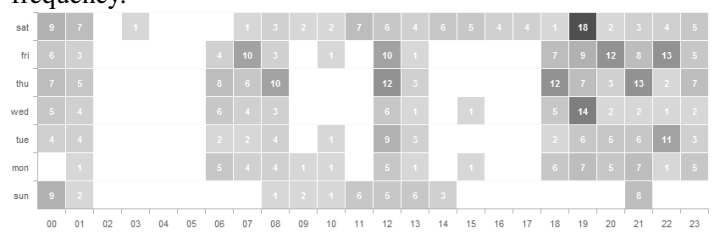

(c) Horizontal axis: hour of the day. Vertical axis: day of the week. Darker blocks indicate periods with higher broadcast frequency.

Fig. 11. Visualization of information related to Daihatsu Tanto Exe TV commercial.

[14] S.-A. Berrani, G. Manson, and P. Lechat, "A non-supervised approach for repeated sequence detection in tv broadcast streams," Image Commun., vol. 23, no. 7, pp. 525-537, August 2008.

[15] I. Döhring and R. Lienhart, "Mining tv broadcasts for recurring video sequences," in Proceeding of the ACM International Conference on Image and Video Retrieval, 2009, pp. 1-8.

[16] N. Putpuek, N. Cooharojananone, C. Lursinsap, and S. Satoh, "Unified approach to detection and identification of commercial films by temporal occurrence pattern," in 2010 20th International Conference on Pattern Recognition (ICPR), Aug. 2010, pp. 3288-3291.

[17] J. Haitsma and T. Kalker, "A highly robust audio fingerprinting system," in 3rd International Conference on Music Information Retrieval, 2002.

[18] S. Poullot, M. Crucianu, and O. Buisson, "Scalable mining of large video databases using copy detection," in Proceeding of the 16th ACM international conference on Multimedia, 2008, pp. 61-70.

[19] S. Poullot, M. Crucianu, and S. Satoh, "Indexing local configurations of features for scalable content-based video copy detection," in Proceedings of the First ACM workshop on Large-scale multimedia retrieval and mining, 2009, pp. 43-50.

[20] H. Jégou, M. Douze, and C. Schmid, "Improving bag-of-features for large scale image search," Int. J. Comput. Vision, vol. 87, no. 3, pp. 316-336, May 2010. 
[21] W.-L. Zhao, X. Wu, and C.-W. Ngo, "On the annotation of web videos by efficient near-duplicate search," IEEE Transactions on Multimedia, vol. 12, no. 5, pp. 448-461, Aug. 2010.

[22] X. Wu, M. Takimoto, S. Satoh, and J. Adachi, "Scene duplicate detection based on the pattern of discontinuities in feature point trajectories," in Proceeding of the 16th ACM international conference on Multimedia, 2008, pp. 51-60.

[23] H.-K. Tan, C.-W. Ngo, R. Hong, and T.-S. Chua, "Scalable detection of partial near-duplicate videos by visual-temporal consistency," in Proceedings of the seventeen ACM international conference on Multimedia, 2009, pp. 145-154.

[24] L. Shang, L. Yang, F. Wang, K.-P. Chan, and X.-S. Hua, "Real-time large scale near-duplicate web video retrieval," in Proceedings of the international conference on Multimedia, 2010, pp. 531-540.

[25] X. Zhou and L. Chen, "Monitoring near duplicates over video streams," in Proceedings of the international conference on Multimedia, 2010, pp. 521-530.

[26] Trec video retrieval evaluation home page. [Online]. Available: http://www-nlpir.nist.gov/projects/trecvid/

[27] S. Satoh, "Simple low-dimensional features approximating ncc-based image matching," Pattern Recognition Letters, vol. 32, no. 14, pp. 1902 1911, 2011.

[28] P. Cano, E. Batlle, T. Kalker, and J. Haitsma, "A review of audio fingerprinting," J. VLSI Signal Process. Syst., vol. 41, no. 3, pp. 271284, November 2005 